\title{
A Review of Cognitive Changes During Acute Aerobic Exercise
}

\begin{abstract}
Julie A. Cantelon ${ }^{1,2,3 *}$ and Grace E. Giles ${ }^{1,2}$
' U.S. Army Combat Capabilities Development Command Soldier Center, Natick, MA, United States, ${ }^{2}$ Center for Applied Brain and Cognitive Sciences, Tufts University, Medford, MA, United States, ${ }^{3}$ Department of Psychology, Tufts University, Medford, MA, United States
\end{abstract}

A growing body of work has investigated the effects of acute, or single bouts of, aerobic exercise on cognitive function. However, review of this research has largely focused on changes following exercise, with less focus on cognitive changes during exercise. The purpose of this review is to discuss the critical characteristics of this literature to date, including: (1) what has been done, (2) what has been found, and (3) what is next. Furthermore, previous meta-analytic reviews have demonstrated there is a small positive effect on cognition when measured during exercise, with executive functions showing the largest effects. However, these reviews group executive functions together. Here we explore how inhibition, working memory and cognitive flexibility are individually impacted by factors such as exercise intensity or duration. Searches of electronic databases and reference lists from relevant studies resulted in 73 studies meeting inclusion criteria. Studies were grouped by executive and non-executive cognitive domains, intensity and duration of exercise bouts. Within the executive domain, we found that effects on working memory and cognitive flexibility remain mixed, effects on inhibition are clearer. Moderate intensity exercise improves response time, vigorous intensity impairs accuracy. Moderate to vigorous intensity improves response time across non-executive domains of attention, motor speed and information processing, with no significant effects on accuracy. Memory processes are consistently improved during exercise. Effects of exercise duration on response time and accuracy are nuanced and vary by cognitive domain. Studies typically explore durations of 45 min or less, extended exercise durations remain largely unexplored. We highlight factors to consider when assessing exercise-cognition relationships, as well as current gaps and future directions for work in this field.

Keywords: exercise, cognition, executive function, physical activity, review

\section{INTRODUCTION}

Over the past decade research examining the relationship between exercise and cognition has grown tremendously. It is now widely accepted that long-term, or chronic, exercise can benefit both mental and physical health, including brain and cognitive function (Piercy et al., 2018; Erickson et al., 2019). More recently, research has begun to examine the effects of acute, or single bouts, of exercise on cognitive function. The majority of previous work examining such acute effects have compared cognitive changes before and after bouts of physical activity, with less focus on changes evoked 
during physical activity. However, understanding how physical activity, or "exercise," as it is often referred to colloquially, impacts cognitive function is critical in a number of applied settings. For instance, individuals with high-stress jobs (e.g., law enforcement and military personnel) often operate under physically demanding conditions while making critical, life or death decisions. Furthermore, athletes are tasked with decisionmaking, pacing strategies, and emotion regulation during competition (Hyland-Monks et al., 2018).

Research has begun to demonstrate how cognition is impacted during exercise. Indeed, previous meta-analytic and integrative reviews have identified several moderating variables influencing cognitive changes during exercise. Specifically, cognitive domain, exercise intensity, duration, modality and an individual's fitness level have all been shown to influence cognitive performance (McMorris and Graydon, 2000; Brisswalter et al., 2002; Tomporowski, 2003; Lambourne and Tomporowski, 2010; Dietrich and Audiffren, 2011; Chang et al., 2012; Davranche et al., 2015). Furthermore, timing of cognitive task administration also impacts the direction of effects. For example, task administration in the initial $20 \mathrm{~min}$ of exercise has been shown to result in detrimental or negligible effects, whereas task administration after $20 \mathrm{~min}$ of exercise results in beneficial effects. Finally, tasks categorized as measures of executive function show beneficial and significantly larger effects than any other domain of cognitive tasks (e.g., information processing, simple and choice reaction time, attention, memory) (Lambourne and Tomporowski, 2010; Chang et al., 2012).

Despite heterogeneity in empirical findings, there are clear theoretical predictions to explain changes in cognitive function during acute exercise. Early conceptualizations of acute exercisecognition interactions were built upon arousal-performance theories (Yerkes and Dodson, 1908; Easterbrook, 1959). These posit that exercise-induced increases in physiological arousal and catecholamine activity (e.g., dopamine and norepinephrine) alter cognitive performance in an inverted- $U$ pattern (McMorris et al., 2011; McMorris and Hale, 2012). For instance, improved memory in particular has been associated with increased dopamine and norepinephrine, as well as brain derived neurotrophic factor (BDNF) (Roig et al., 2013). Here, moderate-intensity exercise would enhance cognitive performance more than low- or high-intensity exercise. This early work largely focused on lower level sensory and perceptual processes, such as motor speed and information processing (McMorris and Graydon, 2000; Tomporowski, 2003). However, it has been argued that higher-order cognitive processes are more likely to be affected by exercise, particularly when assessed during exercise (McMorris and Graydon, 2000; Dietrich, 2003). These processes involve executive functions, also termed cognitive control, and include inhibition, working memory, and cognitive flexibility (Miyake et al., 2000). The Transient Hypofrontality Theory (THT) was developed to explain exercise effects on these higher-order processes. The THT holds that exercise recruits activity in motor pathways (e.g., primary and secondary motor cortices, basal ganglia, cerebellum) as well as sensory (e.g., primary sensory cortex) and autonomic pathways (e.g., hypothalamus) at the expense of structures supporting higher-order cognitive processing, including the prefrontal cortex (PFC) (Dietrich, 2006). The more recent Reticular-Activating Hypofrontality (RAH) model expands on this concept, stating that exercise activates the arousal-related reticular activating system, which benefits cognition up to certain exercise intensities, at which point exercise deactivates the prefrontal cortex leading to impairments in executive function (Dietrich and Audiffren, 2011). Taken together, current theories suggest that increasing exercise intensity or duration enhances or sustains performance on sensory and motor tasks, but impairs executive control processes.

Although previous empirical work and reviews have advanced our understanding of exercise-cognition relationships, many questions still remain. Given the complexities in both cognitive functioning and the physiological processes involved in physical exercise, it is unsurprising that the literature in this field varies greatly. Moreover, more than 30 studies have been published since the last comprehensive review of this field. We believe an updated discussion of the critical characteristics of this literature, as well as specific avenues for future research, may promote advancements both by those working within this field, or others new to this area. Therefore, in this review we aim to answer the following questions: (1) what has been done? (2) what has been found? (3) what is next?

\section{WHAT HAS BEEN DONE?}

\section{Gathering Extant Literature}

In this review we will explore extant research examining cognitive changes during acute exercise (see Pontifex et al., 2019 for review of cognitive changes following acute exercise). More specifically, this review is limited to studies that employed a bout of aerobic exercise and collected measures of cognitive performance while the participant was in the process of engaging in physical activity. Additionally, we restricted inclusion to studies manipulating exercise load using intensity or duration and excluded studies using physical load manipulations (i.e., load carriage). The review is limited to studies using healthy young adult populations. To identify studies eligible for this review, a computerized search for studies in Google Scholar and Pubmed (through May 30, 2021) was conducted using the following keywords: searches used the logical operator "OR" between exercise-related terms (i.e., "exercise," "physical exercise," "physical activity") and the logical operator "AND" between the exercise-related terms and either the cognition search modifier cogniti* (i.e., "cognition," "cognitive"), or cognitive tasks previously identified in reviews of this field (i.e., "stroop task") (see Pontifex et al., 2019 for list of cognitive tasks). Unpublished and non-peer reviewed studies were excluded. This search strategy resulted in a total of 73 studies that investigated cognition during acute exercise. Studies were then classified by authors (JC and GG) with regards to sample size, characteristics of subject population, exercise intensity and duration, executive and non-executive cognitive domain and cognitive task, main findings (categorization provided in Tables 1-7). Study design, participant fitness level, exercise 
modality and specific timing of cognitive testing during exercise are provided in Supplementary Table 1 . To note, all studies that met inclusion criteria used exercise modes of cycling ( $80 \%$ of studies), running or walking (20\% of studies), with one study using kayak ergometry (Davranche et al., 2009).

Studies were also characterized with regards to the intensity and duration of the exercise bout (see Table $\mathbf{8}$ for classification criteria). Exercise intensity relates to how much energy is expended, or how hard your body is working during exercise. Common methods for prescribing and monitoring intensity during aerobic exercise include percentage of maximum heart rate (HRmax), heart rate reserve (HRR), maximal oxygen uptake $\left(\mathrm{VO}_{2} \mathrm{max}\right)$, or oxygen uptake reserve $\left(\mathrm{VO}_{2}\right.$ reserve). Studies using these methods to prescribe relative intensity (i.e., energy cost in relation to an individual's max capacity) were then classified into intensity categories using criteria from American College of Sports Medicine guidelines for Exercise Testing and Prescription (Table 6.1; Garber et al., 2011). Intensity categories included very-light (i.e., $<37 \% \mathrm{VO}_{2} \max$ or $<57 \% \mathrm{HRmax}$ ), light (37$45 \% \mathrm{VO}_{2} \max$ or $57-63 \% \mathrm{HRmax}$ ), moderate (45-63\% $\mathrm{VO}_{2} \max$ or $64-76 \% \mathrm{HRmax})$, vigorous (64-90\% $\mathrm{VO}_{2} \max$ or $77-95 \%$ HRmax) and near-maximal to maximal ( $\geq 91 \% \mathrm{VO}_{2} \max$ or $\geq 96 \% \mathrm{HRmax}$ ). If maximum power output ( $W \max$ ) values were reported, conversion formulas were used to obtain $\% \mathrm{V0}_{2} \max$ (Arts and Kuipers, 1994). Additionally, we explored whether these effects were moderated by factors such as characteristics of the participants (i.e., fitness level, sports expertise), exercise protocol (intensity, duration, mode), and dependent outcome measure (response time, accuracy).

\section{What Aspects of Cognition Have Been Investigated?}

There are many ways one might characterize the types of cognitive functions that have been investigated during acute aerobic exercise. In this review, we classified the tasks used in each study by the executive or non-executive cognitive domain assessed (see Supplementary Table $\mathbf{1}$ for classification scheme). To date, executive function is the most extensively studied cognitive domain, comprising more than $45 \%$ of the total extant literature examining cognitive function during acute aerobic exercise. Therefore, we will primarily focus on the work that has been conducted within this domain, but will also briefly review other non-executive domains, including attention, motor speed, information processing, and memory.

\section{Executive Functions}

Executive functions, also referred to as cognitive control processes, are often grouped together as they all facilitate goaldirected behaviors and rely on a similar fronto-cingulo-parietal network (Alvarez and Emory, 2006). However, previous work has demonstrated that they are separable functions, including mental set shifting (moving back and forth between tasks, also termed cognitive flexibility), information updating (integrating new information, also termed working memory), and inhibition (withholding a prepotent response) (Miyake et al., 2000; Miyake and Friedman, 2012).
Although previous meta-analytic reviews have reported positive effect sizes when executive functions are measured during exercise, these conclusions are largely drawn from work examining inhibition (comprising $32 \%$ of all experiments measuring executive functions during exercise). As such, individual empirical studies examining single or separate executive functions have not consistently reported improved performance during exercise. Specifically, recent work has demonstrated that during exercise inhibitory control improves, working memory declines and effects on cognitive flexibility are dependent upon the specific task used to assess performance (Ando et al., 2014; Oppezzo and Schwartz, 2014). Thus, the effects on executive functions during exercise appear to be specific and dependent on several moderating variables (Dietrich and Audiffren, 2011; Audiffren, 2016). The domain-general effects often reported when cognition is measured following exercise may not necessarily be generalized across differing cognitive domains when cognition is measured during exercise. Therefore, here we will examine how separable executive functions of inhibition, working memory and cognitive flexibility have been studied and are impacted during exercise.

\section{Inhibition}

Inhibition (also referred to as "inhibitory control") refers to the ability to suppress goal-irrelevant stimuli or behavioral responses. Inhibition can be dissociated into motor response inhibition and interference control (also termed cognitive inhibition) (Friedman and Miyake, 2004; Diamond, 2012; Nigg, 2017). Motor response inhibition involves inhibition of prepotent and automatic motor responses. Motor response inhibition has commonly been measured during exercise using non-selective stopping tasks, such as the Go No/Go Task (Donders, 1969) or Stop-Signal task (Logan and Cowan, 1984). Interference control refers to the ability to resist interference from goal-irrelevant stimuli within the environment. Interference control during exercise has been measured using stimulus-response compatibility tasks, such as the Stroop Test (Stroop, 1935), Eriksen Flanker Task (Eriksen and Eriksen, 1974), Simon Task (Simon and Wolf, 1963), or Attention Network Task (ANT)- Executive (Fan et al., 2002; see Table 1).

\section{Working Memory}

Working memory (also referred to as updating) refers to the ability to temporarily store and manipulate information (Miyake and Shah, 1999). Measures of working memory utilized during acute aerobic exercise include the n-back task, spatial delayed-response task (DR), Sternberg task, or random number generation task (RNG) (see Table 2).

\section{Cognitive Flexibility}

Cognitive flexibility (also referred to as "shifting") refers to the ability to switch between different mental sets, tasks, or strategies (Miyake and Friedman, 2012). A wide range of tasks have been used to assess varying aspects of cognitive flexibility during exercise. Majority of studies have used the Wisconsin Card Sorting Task (WCST) to assess perseverance and set-shifting, or the local global task to assess switching between local and global attentional processing. Studies have also used Guilfords' alternate uses task (GAU) and the compound remote associates task (CRA) 
TABLE 1 | Summary of studies examining inhibition during acute aerobic exercise.

\begin{tabular}{|c|c|c|c|c|c|c|c|c|c|}
\hline \multicolumn{3}{|c|}{ Sample characteristics } & \multirow[b]{2}{*}{ Prescribed intensity } & \multicolumn{3}{|c|}{ Exercise } & \multicolumn{2}{|c|}{ Main results } & \multirow[b]{2}{*}{$\begin{array}{l}\text { Did performance } \\
\text { improve? }\end{array}$} \\
\hline $\begin{array}{l}\text { Study } \\
\text { (year) }\end{array}$ & $n(\mathrm{~F})$ & Age & & Intensity category & Duration $^{a}$ & Cognitive task & Response time & Accuracy & \\
\hline $\begin{array}{l}\text { John et al. } \\
\text { (2009) }\end{array}$ & $20(9)$ & $\begin{array}{l}26.4 \pm 4.04 \\
\text { years }\end{array}$ & Rest, $1 \mathrm{MPH}$ & Rest, very light & $N R$ & Stroop & No differences & No differences & No effects \\
\hline $\begin{array}{l}\text { Davranche } \\
\text { et al. (2009) }\end{array}$ & $14(3)$ & $30 \pm 8$ years & Rest, 50\% MAP & Rest, moderate & $\begin{array}{l}215 \text {-min } \\
\text { Periods ( } 5 \text { min } \\
\text { rest between) }\end{array}$ & Eriksen flanker & Moderate < rest & No differences & Yes; only RT \\
\hline $\begin{array}{l}\text { Ando et al. } \\
\text { (2011) }\end{array}$ & $12(0)$ & $\begin{array}{c}25.3 \pm 3.1 \\
\text { years }\end{array}$ & $\begin{array}{l}\text { Rest, } 40,60,80 \% \\
\mathrm{VO}_{2} \text { peak }\end{array}$ & $\begin{array}{l}\text { Rest, light, } \\
\text { moderate, vigorous }\end{array}$ & $6.5 \mathrm{~min}$ & Eriksen flanker & $\begin{array}{l}\text { Overall RT (motor time): No } \\
\text { differences; pre-motor time: } \\
\text { moderate < rest }\end{array}$ & Error rate: rest < vigorous & $\begin{array}{l}\text { No; ACC at } \\
\text { vigorous }\end{array}$ \\
\hline $\begin{array}{l}\text { Labelle } \\
\text { et al. (2013) }\end{array}$ & $37(18)$ & $\begin{array}{l}23.8 \pm 2.6 \\
\text { years }\end{array}$ & 40, 60, 80\% PPO & $\begin{array}{l}\text { Light, moderate, } \\
\text { vigorous }\end{array}$ & $6.5 \mathrm{~min}$ & Modified stroop & No differences & No differences & No effects \\
\hline $\begin{array}{l}\text { Pontifex } \\
\text { and Hillman } \\
\text { (2007) }\end{array}$ & $41(26)$ & $\begin{array}{l}20.2 \pm 1.6 \\
\text { years }\end{array}$ & Rest, 60\% HRmax & Rest, light & $6.5 \mathrm{~min}$ & Eriksen flanker & No differences & $\begin{array}{l}\text { Incongruent accuracy: } \\
\text { light < rest; congruent } \\
\text { accuracy: no differences }\end{array}$ & No; only ACC \\
\hline $\begin{array}{l}\text { Komiyama } \\
\text { et al. (2019) }\end{array}$ & $17(0)$ & $\begin{array}{l}22.1 \pm 1.7 \\
\text { years }\end{array}$ & $\begin{array}{l}\text { Rest, } 50 \%, 80 \% \\
\mathrm{VO}_{2} \text { peak (performed } \\
\text { within } 1 \text { exercise } \\
\text { session) }\end{array}$ & $\begin{array}{l}\text { Rest, moderate, } \\
\text { vigorous }\end{array}$ & $8 \min$ & Go/No-Go & No differences & $\begin{array}{l}\text { Accuracy score: } \\
\text { vigorous < rest }\end{array}$ & $\begin{array}{l}\text { No; only ACC at } \\
\text { vigorous }\end{array}$ \\
\hline $\begin{array}{l}\text { Ando et al. } \\
\text { (2014) }\end{array}$ & $12(0)$ & $\begin{array}{l}22.9 \pm 1.5 \\
\text { years }\end{array}$ & Rest, $60 \% \mathrm{VO}_{2}$ peak & Rest, moderate & $10 \mathrm{~min}$ & Go/No-Go & Moderate < rest & No differences & Yes; only RT \\
\hline $\begin{array}{l}\text { Smith et al. } \\
\text { (2016) }\end{array}$ & $15(9)$ & $28 \pm 5$ years & Rest, 70, 90\% HRR & $\begin{array}{l}\text { Rest, vigorous, } \\
\text { near-maximal }\end{array}$ & $10 \mathrm{~min}$ & Go/No-Go & $\begin{array}{l}\text { Near-maximal > vigorous, } \\
\text { rest }\end{array}$ & $\begin{array}{l}\text { Omission errors: vigorous, } \\
\text { rest < near-maximal; false } \\
\text { alarms: vigorous, } \\
\text { rest < near-maximal }\end{array}$ & $\begin{array}{l}\text { No; RT and ACC at } \\
\text { near-maximal }\end{array}$ \\
\hline $\begin{array}{l}\text { Ando et al. } \\
\text { (2013) }\end{array}$ & $12(0)$ & $\begin{array}{l}22.9 \pm 1.5 \\
\text { years }\end{array}$ & $\begin{array}{l}\text { Rest, } 60 \% \mathrm{VO}_{2} \text { peak } \\
(\sim 80 \% \mathrm{HRmax})\end{array}$ & Rest, vigorous & $\begin{array}{l}10 \mathrm{~min}(5 \mathrm{~min} \\
\text { warm-up) }\end{array}$ & Go/No-Go & Vigorous < rest & No differences & Yes, only RT \\
\hline $\begin{array}{l}\text { Davranche } \\
\text { et al. (2009) }\end{array}$ & $12(4)$ & $18 \pm 4$ years & $\begin{array}{l}40 \pm 4,75 \pm 2 \% \\
\operatorname{HRmax}\end{array}$ & Very light, moderate & $\begin{array}{l}14 \mathrm{Min} \text { (10 } \mathrm{min} \\
\text { warm-up) }\end{array}$ & Simon & Moderate < very light & No differences & $\begin{array}{l}\text { Yes; only RT at } \\
\text { higher intensity }\end{array}$ \\
\hline $\begin{array}{l}\text { Davranche } \\
\text { et al. (2015) }\end{array}$ & $14(3)$ & $21 \pm 2$ years & $\begin{array}{l}V T-20 \%, V T, \\
V T+20 \%(\sim 74,81, \\
90 \% \text { HRmax) }\end{array}$ & Moderate, vigorous & $\begin{array}{l}15 \mathrm{~min}(5 \mathrm{~min} \\
\text { warm-up) }\end{array}$ & Simon & $\begin{array}{l}\text { Min } 10 \text { moderate, } \\
\text { vigorous }<\text { min } 0 \\
\text { moderate, vigorous }\end{array}$ & No differences & Yes; only RT \\
\hline $\begin{array}{l}\text { McMorris } \\
\text { et al. (2009) }\end{array}$ & $24(0)$ & $\begin{array}{l}24.32 \pm 7.10 \\
\quad \text { years }\end{array}$ & Rest, 50, 80\% MAP & $\begin{array}{l}\text { Rest, moderate, } \\
\text { vigorous }\end{array}$ & $\begin{array}{l}15 \min (5 \min \\
\text { warm-up) }\end{array}$ & Eriksen flanker & $\begin{array}{l}\text { Overall RT: moderate, } \\
\text { rest < vigorous; RT } \\
\text { post-correct responses: } \\
\text { moderate, rest < vigorous; } \\
\text { RT post-error responses: } \\
\text { no differences }\end{array}$ & $\begin{array}{l}\text { Errors: moderate, } \\
\text { rest }<\text { vigorous }\end{array}$ & $\begin{array}{l}\text { No; RT and ACC at } \\
\text { vigorous }\end{array}$ \\
\hline
\end{tabular}


TABLE 1 | (Continued)

\begin{tabular}{|c|c|c|c|c|c|c|c|c|c|}
\hline \multicolumn{3}{|c|}{ Sample characteristics } & \multirow[b]{2}{*}{ Prescribed intensity } & \multicolumn{3}{|c|}{ Exercise } & \multicolumn{2}{|c|}{ Main results } & \multirow[b]{2}{*}{$\begin{array}{l}\text { Did performance } \\
\text { improve? }\end{array}$} \\
\hline $\begin{array}{l}\text { Study } \\
\text { (year) }\end{array}$ & $n(\mathrm{~F})$ & Age & & Intensity category & Duration $^{a}$ & Cognitive task & Response time & Accuracy & \\
\hline $\begin{array}{l}\text { Komiyama } \\
\text { et al. (2017) }\end{array}$ & $13(0)$ & $\begin{array}{c}21.5 \pm 3.5 \\
\text { years }\end{array}$ & Rest, $50 \% \mathrm{VO}_{2}$ peak & Rest, moderate & $\begin{array}{l}15 \min (5 \min \\
\text { warm-up) }\end{array}$ & Go/No-Go & Light $<$ Rest & No differences & Yes; only RT \\
\hline $\begin{array}{l}\text { Schmit } \\
\text { et al. (2015) }\end{array}$ & $15(5)$ & $\begin{array}{l}22.1 \pm 0.6 \\
\text { years }\end{array}$ & Rest, 80\% MAP & Rest, vigorous & $\begin{array}{l}20 \mathrm{~min}(3 \mathrm{~min} \\
\text { warm-up) }\end{array}$ & Eriksen flanker & No differences & $\begin{array}{l}\text { Incongruent errors: } \\
\text { rest < vigorous; congruent } \\
\text { errors: no differences }\end{array}$ & No; only ACC \\
\hline $\begin{array}{l}\text { Finkenzeller } \\
\text { et al. (2019) }\end{array}$ & $52(24)$ & $\begin{array}{l}22.12 \pm 2.56 \\
\text { years }\end{array}$ & $\begin{array}{l}\text { Rest, } 30,30+60,60 \% \\
\text { MAP }\end{array}$ & $\begin{array}{l}\text { Rest, very light, } \\
\text { very light/moderate } \\
\text { varied, moderate }\end{array}$ & $\begin{array}{l}20 \min (4 \min \\
\text { warm-up) }\end{array}$ & Eriksen flanker & $\begin{array}{l}\text { Congruent RT: Min } 16 \text { Very } \\
\text { light/moderate < rest; Min } \\
8,12,16 \text { moderate < rest }\end{array}$ & $\begin{array}{l}\text { Incongruent errors: } \\
\text { moderate > rest; congruent } \\
\text { errors: no differences }\end{array}$ & $\begin{array}{l}\text { Yes; RT } \\
\text { No; ACC }\end{array}$ \\
\hline $\begin{array}{l}\text { Davranche } \\
\text { and } \\
\text { McMorris } \\
\text { (2009) }\end{array}$ & $12(4)$ & $32 \pm 9$ years & $\begin{array}{l}\text { Rest, lactate threshold } \\
\text { power ( } 77 \pm 4 \% \\
\text { HRmax) }\end{array}$ & Rest, moderate & $21 \mathrm{Min}$ & Simon & $\begin{array}{l}\text { Overall RT: } \\
\text { moderate < rest; simon } \\
\text { effect: rest < moderate }\end{array}$ & No differences & No; only RT \\
\hline $\begin{array}{l}\text { Joyce et al. } \\
\text { (2009) }\end{array}$ & $10(3)$ & $23 \pm 2$ years & Rest, 40\% MAP & Rest, light & $\begin{array}{l}25 \mathrm{~min}(4 \mathrm{~min} \\
\text { warm-up) }\end{array}$ & Stop-signal & $\begin{array}{l}\text { Go RT: light < rest; } \\
\text { stop-signal RT: light < rest }\end{array}$ & No differences & Yes; only RT \\
\hline $\begin{array}{l}\text { Huertas } \\
\text { et al. (2011) }\end{array}$ & $30(0)$ & $17 \pm 2$ years & Rest, 80, 95\% LT & $\begin{array}{l}\text { Rest, moderate, } \\
\text { vigorous }\end{array}$ & $\begin{array}{l}25 \min (10 \mathrm{~min} \\
\text { warm-up) }\end{array}$ & ANT-I & No differences & No differences & No effects \\
\hline $\begin{array}{l}\text { Olson et al. } \\
\text { (2016) }\end{array}$ & $27(11)$ & $\begin{array}{l}20.4 \pm 2.0 \\
\text { years }\end{array}$ & $\begin{array}{l}\text { Rest, } 40,60 \% \\
\mathrm{VO}_{2} \text { peak }\end{array}$ & $\begin{array}{l}\text { Rest, light, } \\
\text { moderate }\end{array}$ & $\begin{array}{l}26 \min (5 \min \\
\text { warm-up) }\end{array}$ & Eriksen flanker & Moderate < light, rest & $\begin{array}{l}\text { Incongruent accuracy: light, } \\
\text { moderate < rest; congruent } \\
\text { accuracy: no differences }\end{array}$ & $\begin{array}{l}\text { Yes; RT At } \\
\text { moderate: } \\
\text { No; ACC }\end{array}$ \\
\hline $\begin{array}{l}\text { Komiyama } \\
\text { et al. (2015) }\end{array}$ & $16(0)$ & $\begin{array}{l}23.0 \pm 2.3 \\
\text { years }\end{array}$ & 140 bpm & Rest, moderate & $\begin{array}{l}30 \mathrm{~min}(5 \mathrm{~min} \\
\text { warm-up) }\end{array}$ & Go/No-Go & Min 23 moderate < rest & No differences & Yes; only RT \\
\hline $\begin{array}{l}\text { Komiyama } \\
\text { et al. (2016) }\end{array}$ & $10(0)$ & $\begin{array}{l}22.3 \pm 2.1 \\
\text { years }\end{array}$ & $\begin{array}{l}\text { Rest, } 140 \text { bpm ( 71\% } \\
\text { HRmax) }\end{array}$ & Rest, moderate & $\begin{array}{l}30 \min (5 \min \\
\text { warm-up) }\end{array}$ & Go/No-Go & Moderate $<$ rest & $\begin{array}{l}\text { Go accuracy: } \\
\text { rest < moderate; NoGo } \\
\text { accuracy: no differences }\end{array}$ & Yes; RT and ACC \\
\hline $\begin{array}{l}\text { Sanchis } \\
\text { et al. (2020) }\end{array}$ & $24(0)$ & $\begin{array}{l}22.6 \pm 2.9 \\
\text { years }\end{array}$ & $80 \%$ VT1, 80\% VT2 & Light, moderate & $\begin{array}{l}33 \mathrm{~min} 45 \mathrm{~s} \\
\text { (6.5 min } \\
\text { warm-up) }\end{array}$ & $\begin{array}{l}\text { ANTI-I } \\
\text { (Executive) }\end{array}$ & No differences & No differences & No effects \\
\hline $\begin{array}{l}\text { Tempest } \\
\text { et al. (2017) }\end{array}$ & $14(5)$ & $\begin{array}{l}22.7 \pm 3.8 \\
\text { years }\end{array}$ & $\begin{array}{l}<30 \text { Watts, } 10 \% \\
\text { Above VT }\end{array}$ & Very light, vigorous & $60 \mathrm{~min}$ & Eriksen flanker & $\begin{array}{l}\mathrm{RT} \text { : end-vigorous } \\
\text { exercise < beginning- } \\
\text { vigorous } \\
\text { exercise }\end{array}$ & $\begin{array}{l}\text { Incongruent accuracy: end- } \\
\text { very light, end- } \\
\text { vigorous < beginning-very } \\
\text { light, beginning vigorous; } \\
\text { congruent accuracy: no } \\
\text { differences }\end{array}$ & $\begin{array}{l}\text { Yes; RT at higher } \\
\text { intensity } \\
\text { No; ACC }\end{array}$ \\
\hline $\begin{array}{l}\text { Giles et al. } \\
\text { (2018) }\end{array}$ & $36(21)$ & $\begin{array}{l}23.4 \pm 3.6 \\
\text { years }\end{array}$ & 57, $70 \%$ HRmax & Light, moderate & $\begin{array}{l}90 \min (5 \min \\
\text { warm-up) }\end{array}$ & Stroop & $\begin{array}{l}\text { Min } 30 \text { moderate < Min } 30 \\
\text { light; Min } 90 \\
\text { moderate }<\text { Min } 90 \text { light }\end{array}$ & No differences & $\begin{array}{l}\text { Yes; only RT at } \\
\text { higher intensity }\end{array}$ \\
\hline
\end{tabular}

n, number of participants; F, females; "-" = not measured; RT, reaction time; ACC, accuracy; NR, not reported; Simon Effect = Incongruent RT-Congruent RT; Accuracy Score = Correct Responses/Total Responses; ANT-I, attentional network task (inhibition); VO $\mathrm{O}_{2} \max$, maximum volume of oxygen uptake; VO ${ }_{2}$ peak, peak oxygen uptake; HRmax, maximum heart rate; HRR, heart rate reserve; VT, ventilatory threshold; PPO, peak power output; MAP, maximum aerobic power; VAT, ventilatory anaerobic threshold; RPE, rating of perceived exertion.

${ }^{a}$ Exercise duration excludes warm-up time. 
TABLE 2 | Summary of studies examining working memory during acute aerobic exercise.

\begin{tabular}{|c|c|c|c|c|c|c|c|c|c|}
\hline \multicolumn{3}{|c|}{ Sample characteristics } & \multirow[b]{2}{*}{$\begin{array}{l}\text { Prescribed } \\
\text { intensity }\end{array}$} & \multicolumn{3}{|c|}{ Exercise } & \multicolumn{2}{|c|}{ Main results } & \multirow[b]{2}{*}{$\begin{array}{l}\text { Did performance } \\
\text { improve? }\end{array}$} \\
\hline Study (year) & $n(\mathbf{F})$ & Age & & $\begin{array}{l}\text { Intensity } \\
\text { category }\end{array}$ & Duration $^{a}$ & Cognitive task & Response time & Accuracy & \\
\hline $\begin{array}{l}\text { Quelhas } \\
\text { Martins et al. } \\
\text { (2013) } \\
\text { Experiment } 2\end{array}$ & $55(65)$ & $\begin{array}{c}19.57 \pm 0.83 \\
\text { years }\end{array}$ & $\begin{array}{l}\text { Rest (within) and } 5 \text { watts, } \\
50-60 \text { watts, or } 75-90 \\
\text { watts (between) }(41,61 \\
\text { and } 64 \% \text { HRmax) }\end{array}$ & $\begin{array}{l}\text { Rest, very light, } \\
\text { light and moderate }\end{array}$ & $\begin{array}{l}\text { NR: } \sim 20 \text { min } \\
\text { (2 min } \\
\text { warm-up) }\end{array}$ & Sternberg & $\begin{array}{l}\text { Response latency: } \\
\text { light, } \\
\text { moderate < rest }\end{array}$ & - & Yes \\
\hline $\begin{array}{l}\text { Komiyama } \\
\text { et al. (2019) }\end{array}$ & $17(0)$ & $\begin{array}{l}22.1 \pm 1.7 \\
\text { years }\end{array}$ & $\begin{array}{l}\text { Rest, } 50,80 \% \mathrm{VO}_{2} \text { peak } \\
\text { (performed within } 1 \\
\text { exercise session) }\end{array}$ & $\begin{array}{l}\text { Rest, moderate, } \\
\text { vigorous }\end{array}$ & $8 \mathrm{~min}$ & Spatial DR & No differences & $\begin{array}{l}\text { Accuracy: } \\
\text { vigorous < rest }\end{array}$ & No; only ACC \\
\hline $\begin{array}{l}\text { Komiyama } \\
\text { et al. (2017) }\end{array}$ & $13(0)$ & $\begin{array}{c}21.5 \pm 3.5 \\
\text { years }\end{array}$ & Rest, $50 \% \mathrm{VO}_{2}$ peak & Rest, moderate & $\begin{array}{l}15 \mathrm{~min}(5 \mathrm{~min} \\
\text { warm-up) }\end{array}$ & Spatial DR & No differences & No differences & No effects \\
\hline $\begin{array}{l}\text { Dutke et al. } \\
\text { (2014) }\end{array}$ & $60(14)$ & $\begin{array}{c}M=26.1 \\
\text { years }\end{array}$ & $\begin{array}{l}\text { 75, 120\% VT ( 53-73, } \\
76-95 \% \text { HRmax) }\end{array}$ & $\begin{array}{l}\text { Light-moderate, } \\
\text { vigorous }\end{array}$ & $\begin{array}{l}\sim 27 \mathrm{~min} \\
\text { (10 min } \\
\text { warm-up) }\end{array}$ & $\begin{array}{l}\text { Word } \\
\text { comparison } \\
\text { task (with } \\
\text { button press } \\
\text { every } 2 \text { s) }\end{array}$ & No differences & No differences & No effects \\
\hline $\begin{array}{l}\text { Komiyama } \\
\text { et al. (2016) }\end{array}$ & $10(0)$ & $\begin{array}{c}22.3 \pm 2.1 \\
\text { years }\end{array}$ & $\begin{array}{l}\text { Rest, } 140 \mathrm{bpm}(\sim 71 \% \\
\text { HRmax) }\end{array}$ & Rest, moderate & $\begin{array}{l}30 \mathrm{~min}(5 \mathrm{~min} \\
\text { warm-up) }\end{array}$ & Spatial DR & No differences & No differences & No effects \\
\hline $\begin{array}{l}\text { Komiyama } \\
\text { et al. (2015) }\end{array}$ & $16(0)$ & $\begin{array}{c}23.0 \pm 2.3 \\
\text { years }\end{array}$ & 140 bpm ( 71\% HRmax) & Rest, moderate & $\begin{array}{l}30 \mathrm{~min}(5 \mathrm{~min} \\
\text { warm-up) }\end{array}$ & Spatial DR & No differences & No differences & No effects \\
\hline $\begin{array}{l}\text { Travlos and } \\
\text { Marisi (1995) }\end{array}$ & $\begin{array}{l}20(0) ; 10 \\
\text { low fit, } 10 \\
\text { high fit }\end{array}$ & $\begin{array}{c}23.3 \pm 3.8 \\
\text { years }\end{array}$ & $\begin{array}{l}40,50,60,70,80 \% \\
\mathrm{VO}_{2} \max \end{array}$ & $\begin{array}{l}\text { Light, moderate, } \\
\text { vigorous, }\end{array}$ & $60 \mathrm{~min}$ & RNG & - & No differences & No effects \\
\hline $\begin{array}{l}\text { Rattray and } \\
\text { Smee (2016) }\end{array}$ & $20(10)$ & $\begin{array}{l}\text { Males: } \\
26.6 \pm 5.2 ; \\
\text { Females: } \\
24.6 \pm 5.6 \\
\text { years }\end{array}$ & $\begin{array}{l}\text { Rest, } 90 \% \mathrm{VT} \text { (moderate } \\
\text { constant), } 40 \text { min at } 90 \% \\
\mathrm{VT} \text { then two } 3-\text { min intervals } \\
\text { at } 90 \text { then } 50 \% \mathrm{VO}_{2} \text { max } \\
\text { (moderate varied), } 40 \text { min } \\
\text { at } 90 \% \mathrm{VT} \text { then two } 3 \text {-min } \\
\text { intervals at } 50 \text { then } 90 \% \\
\mathrm{VO}_{2} \text { max } \\
\text { (moderate-vigorous varied) }\end{array}$ & $\begin{array}{l}\text { Rest, moderate } \\
\text { constant, moderate } \\
\text { varied, } \\
\text { moderate-vigorous } \\
\text { varied }\end{array}$ & $60 \mathrm{~min}$ & Speed match & $\begin{array}{l}\text { RT: moderate } \\
\text { constant, } \\
\text { moderate-vigorous } \\
\text { varied < rest }\end{array}$ & No differences & Yes, only RT \\
\hline $\begin{array}{l}\text { Tempest } \\
\text { et al. (2017) }\end{array}$ & $14(5)$ & $\begin{array}{l}22.7 \pm 3.8 \\
\text { years }\end{array}$ & $<30$ watts; $10 \%$ above $\mathrm{VT}$ & Very light, vigorous & $60 \mathrm{~min}$ & 2-Back Task & - & $\begin{array}{l}d^{\prime} \text { : end-vigorous < } \\
\text { beginning-vigorous }\end{array}$ & $\begin{array}{l}\text { No, only ACC at } \\
\text { higher intensity }\end{array}$ \\
\hline
\end{tabular}

n, number of participants; $F$, females; $M$, mean; "-" = not measured; $R T$, reaction time; $A C C$, accuracy; NR, not reported; DR, delayed response task; $R N G$, Random Number Generation; VO 2 max, maximum volume of oxygen uptake; $\mathrm{VO}_{2}$ peak, peak oxygen uptake; HRmax, maximum heart rate $V T$, ventilatory threshold.

a Exercise duration excludes warm-up time. 
to assess convergent and divergent thinking, and one study used a modified version of the Stroop task to assess task-switching (see Table 3).

\section{Non-executive Functions Attention}

Moving beyond executive functions, research has also examined attention during exercise. Attention refers to the ability to selectively focus on relevant information while ignoring other perceivable information (Chun, 2000). Selective attention requires attending to relevant and ignoring non-relevant information, with common tasks used during exercise including odd-ball tasks (Herrmann and Knight, 2001) and local global tasks measuring focus of attention (Navon, 1977). Vigilance is the ability to sustain attention over time and measures used during exercise include continuous performance tasks (CPT), paced auditory serial addition tasks (PASAT), and psychomotor vigilance tasks (PVT) measuring sustained attention and vigilance (Gronwall, 1977; Dinges and Powell, 1985). Last, the Attention Network Task (ANT) has also been used to measure alerting (vigilance), orienting (selection), and executive control of attention (Fan et al., 2002; see Table 4).

\section{Motor Speed}

Motor speed includes several basic elements of motor activity, such as fine motor abilities (dexterity and speed) and reaction time (Harvey, 2019). Majority of studies have employed simple response time (SRT) and choice response time (CRT) tasks to evaluate reaction time during exercise (see Table 5). In a SRT there is a single stimulus and response type, whereas in a CRT there are multiple stimuli each requiring a different response type.

\section{Information Processing}

Information processing refers to the speed and accuracy of processing incoming information (Lichtenberger and Kaufman, 2009). A range of tasks have been used, such as Critical Flicker Fusion (CFF) and visual detection and visual search tasks (see Table 6).

\section{Memory}

Learning refers to a change in behavior resulting from experience, and memory refers to retaining and retrieving that information across the processes of encoding, storage, and retrieval (Crowder, 2015). The present review includes studies in which encoding occurred before or during exercise, such that at least one of three memory processes occurred during exercise. Memory tasks include cued, free or multiple choice recall tasks, prospective memory tasks and map recognition tasks (see Table 7). Given the various timing of when memory processes can be examined during exercise, this domain employs the widest variety of study designs, using both within and between-subjects designs.

\section{WHAT HAS BEEN FOUND?}

Generally, the effects on cognitive performance during acute bouts of exercise are nuanced and dependent on the specific cognitive domain being assessed, as well as the intensity and duration of exercise. To add to the complexity of exercisecognition interactions, effects of intensity and duration are often hard to disentangle due to their inherent physiological connections. However, intensity or duration of exercise may have differing implications and relevance depending on the particular population of interest or research question. Therefore, next we characterize findings by intensity and then by duration. Additionally, we outline research findings in terms of the behavioral dependent variables of response time and accuracy, since previous work has demonstrated that these variables may be differentially impacted by both intensity and duration (McMorris and Hale, 2012).

\section{Findings by Exercise Intensity The Impact of Exercise Intensity on Executive Functions \\ Inhibition}

Inhibition is the most extensively studied cognitive function within the field (comprising over $30 \%$ of the research on cognition during exercise and over $70 \%$ of the research on executive functions). More specifically, the majority of these studies examine inhibition at moderate intensity compared to rest. This breadth allows for a more in depth discussion of commonly observed trends, as well as mixed findings for this particular cognitive function. In general, acute exercise exerts variable effects on inhibitory control, yet certain trends have emerged which warrant further investigation (see Table 1). For example, exercise most often speeds response time for both interference control and motor response inhibition tasks, during moderate intensity, and exercise durations between 0 and $30 \mathrm{~min}$. This beneficial effect of increased response time (without change in accuracy) has been widely observed in the literature and may be attributed to improved efficiency of peripheral motor processes during moderate exercise (Davranche et al., 2005, 2006). Another notable trend is that exercise most often reduces accuracy through increased error rates at increasing exercise intensities (moderate-to-vigorous) and during shorter durations (less than $20 \mathrm{~min}$ ). This reduction in accuracy is primarily driven by false alarm rates, or incongruent errors, on trials requiring greatest amounts of executive control to engage in goaldirected behavior.

Below we examine the effects of specific exercise intensities compared to rest conditions (studies examining inhibition between exercise intensities are discussed in section "Findings by Exercise Duration"). We find that very light and light intensities exert variable effects on inhibitory control. Relative to rest, three studies demonstrated improved response times (Joyce et al., 2009; Komiyama et al., 2017; Finkenzeller et al., 2019) at very light and light intensity, and two demonstrated no effects (John et al., 2009; Ando et al., 2011). With regards to accuracy, two studies demonstrated decreased response accuracy for incongruent trials on the Eriksen Flanker task, with no changes to response time (Pontifex and Hillman, 2007; Olson et al., 2016). Taken together, very light and light intensity exercise may improve speed of motor response inhibition, but impair interference control. However, 
TABLE 3 | Summary of studies examining cognitive flexibility during acute aerobic exercise.

\begin{tabular}{|c|c|c|c|c|c|c|c|c|c|}
\hline \multicolumn{3}{|c|}{ Sample characteristic } & \multirow[b]{2}{*}{$\begin{array}{l}\text { Prescribed } \\
\text { intensity }\end{array}$} & \multicolumn{3}{|c|}{ Exercise } & \multicolumn{2}{|c|}{ Main results } & \multirow[b]{2}{*}{$\begin{array}{l}\text { Did performance } \\
\text { improve? }\end{array}$} \\
\hline Study (year) & $n(\mathrm{~F})$ & Age & & $\begin{array}{l}\text { Intensity } \\
\text { category }\end{array}$ & Duration $^{a}$ & Cognitive task & Response time & Accuracy & \\
\hline $\begin{array}{l}\text { Oppezzo and } \\
\text { Schwartz } \\
\text { (2014) } \\
\text { Experiment } 1\end{array}$ & 48 (NR) & NR & $\begin{array}{l}\text { Rest, } \\
\text { self-selected } \\
\text { walking at } \\
\text { "comfortable" } \\
\text { pace }\end{array}$ & $\begin{array}{l}\text { Rest, very } \\
\text { light-light }\end{array}$ & NR & GAU; CRA & - & $\begin{array}{l}\text { GAU creative appropriate } \\
\text { uses: rest < very light-light; } \\
\text { CRA number of correct } \\
\text { responses: very } \\
\text { light-light < rest }\end{array}$ & Yes; no \\
\hline $\begin{array}{l}\text { Oppezzo and } \\
\text { Schwartz } \\
\text { (2014) } \\
\text { Experiment } 2\end{array}$ & $48(N R)^{b}$ & NR & $\begin{array}{l}\text { Rest, } \\
\text { self-selected } \\
\text { walking at } \\
\text { "comfortable" } \\
\text { pace }\end{array}$ & $\begin{array}{l}\text { Rest, very light- } \\
\text { light }\end{array}$ & $N R$ & GAU & - & $\begin{array}{l}\text { Creative appropriate uses: } \\
\text { rest < very light-light }\end{array}$ & Yes \\
\hline $\begin{array}{l}\text { Pesce et al. } \\
\text { (2003) }\end{array}$ & $16(8)$ & 19-40 years & $\begin{array}{l}\text { Rest, } 60 \% \\
\mathrm{VO}_{2} \max \end{array}$ & Rest, moderate & NR & $\begin{array}{l}\text { Local global task with } \\
\text { switching condition }\end{array}$ & $\begin{array}{l}\text { Overall RT: } \\
\text { moderate < rest; switch } \\
\text { cost: moderate < rest }\end{array}$ & No differences & Yes; only RT \\
\hline $\begin{array}{l}\text { Pesce et al. } \\
\text { (2007) } \\
\text { Experiment } 1\end{array}$ & $\begin{array}{l}24(0) \text { Elite } \\
\text { soccer players, } \\
24(0) \text { Physically } \\
\text { active controls }\end{array}$ & $\begin{array}{l}17.9 \pm 0.8 \\
\text { years }\end{array}$ & Rest, 60\% HRR & Rest, vigorous & NR & $\begin{array}{l}\text { Local global task with } \\
\text { switching condition }\end{array}$ & $\begin{array}{l}\text { Overall RT: non-athletes: } \\
\text { vigorous < rest; athletes: } \\
\text { no differences; switch cost: } \\
\text { non-athletes: } \\
\text { vigorous < rest, athletes: } \\
\text { no differences }\end{array}$ & - & $\begin{array}{l}\text { Yes; only RT in } \\
\text { non-athletes }\end{array}$ \\
\hline $\begin{array}{l}\text { Pesce et al. } \\
\text { (2007) } \\
\text { Experiment } 2\end{array}$ & $\begin{array}{l}\text { Same } \\
\text { participants } \\
\text { and design } \\
\text { as Exp } 1\end{array}$ & & Rest, 60\% HRR & Rest, vigorous & NR & $\begin{array}{l}\text { Local global task with } \\
\text { switching condition }\end{array}$ & $\begin{array}{l}\text { Overall RT: vigorous < rest; } \\
\text { RT switch cost: athletes: } \\
\text { vigorous < rest, } \\
\text { non-athletes: no differences }\end{array}$ & - & $\begin{array}{l}\text { Yes; only RT in } \\
\text { athletes }\end{array}$ \\
\hline $\begin{array}{l}\text { Labelle et al. } \\
\text { (2013) }\end{array}$ & $37(18)$ & $\begin{array}{l}23.8 \pm 2.6 \\
\text { years }\end{array}$ & $\begin{array}{l}40,60,80 \% \\
\text { PPO } \\
\text { (performed } \\
\text { within } 1 \\
\text { exercise } \\
\text { session) }\end{array}$ & $\begin{array}{l}\text { Light, } \\
\text { moderate, } \\
\text { vigorous }\end{array}$ & $6.5 \mathrm{~min}$ & $\begin{array}{l}\text { Modified Stroop with } \\
\text { switching condition }\end{array}$ & No differences & $\begin{array}{l}\text { Switching error rate: light, } \\
\text { moderate < vigorous; } \\
\text { reading non-switch error } \\
\text { rate: light, moderate < } \\
\text { vigorous; inhibition } \\
\text { non-switch error rate: no } \\
\text { differences }\end{array}$ & $\begin{array}{l}\text { No; only ACC at } \\
\text { vigorous }\end{array}$ \\
\hline $\begin{array}{l}\text { Del Giorno } \\
\text { et al. (2010) }\end{array}$ & $30(13)$ & $\begin{array}{c}20.2 \pm 1.1 \\
\text { years }\end{array}$ & $\begin{array}{l}\text { Rest, } 75 \% \text { VT, } \\
\text { VT }\end{array}$ & $\begin{array}{l}\text { Rest, light, } \\
\text { vigorous }\end{array}$ & $\begin{array}{l}25 \text { min (5-min } \\
\text { warm-up) }\end{array}$ & WCST & - & $\begin{array}{l}\text { Unique errors: rest < light, } \\
\text { vigorous; perseverative } \\
\text { errors: no differences; total } \\
\text { number of errors: no } \\
\text { differences }\end{array}$ & No \\
\hline
\end{tabular}


TABLE 3 | (Continued)

\begin{tabular}{|c|c|c|c|c|c|c|c|c|c|}
\hline \multicolumn{3}{|c|}{ Sample characteristic } & \multirow[b]{2}{*}{$\begin{array}{l}\text { Prescribed } \\
\text { intensity }\end{array}$} & \multicolumn{3}{|c|}{ Exercise } & \multicolumn{2}{|c|}{ Main results } & \multirow[b]{2}{*}{$\begin{array}{l}\text { Did performance } \\
\text { improve? }\end{array}$} \\
\hline Study (year) & $n(\mathrm{~F})$ & Age & & $\begin{array}{l}\text { Intensity } \\
\text { category }\end{array}$ & Duration $^{a}$ & Cognitive task & Response time & Accuracy & \\
\hline $\begin{array}{l}\text { Wang et al. } \\
\text { (2013) }\end{array}$ & $80(31)$ & $\begin{array}{c}20.51 \pm 1.99 \\
\text { years }\end{array}$ & $\begin{array}{l}\text { Rest, 30, 50, } \\
80 \% \text { HRR }\end{array}$ & $\begin{array}{l}\text { Rest, light, } \\
\text { moderate, } \\
\text { vigorous }\end{array}$ & $\begin{array}{l}\sim 30 \mathrm{~min} \text { (6 min } \\
\text { warm-up) }\end{array}$ & WCST & - & $\begin{array}{l}\text { Perseverative errors: } \\
\text { moderate, light, } \\
\text { rest < vigorous; correct } \\
\text { conceptual-level } \\
\text { responses: } \\
\text { vigorous < moderate, light, } \\
\text { rest; number of categories } \\
\text { completed: } \\
\text { vigorous < moderate, light, } \\
\text { rest; failure to maintain set: } \\
\text { no differences }\end{array}$ & $\begin{array}{l}\text { No; only ACC at } \\
\text { vigorous }\end{array}$ \\
\hline $\begin{array}{l}\text { Dietrich and } \\
\text { Sparling } \\
(2004)\end{array}$ & $24(0)^{b}$ & $\begin{array}{c}23.7 \pm 9.4 \\
\text { years }\end{array}$ & $\begin{array}{l}\text { Rest, } 70-80 \% \\
\text { HRmax ( 140- } \\
160 \text { bpm) } \\
\text { running or } \\
\text { cycling }\end{array}$ & $\begin{array}{l}\text { Rest, } \\
\text { moderate- } \\
\text { vigorous }\end{array}$ & $\begin{array}{l}45 \text { min (5-min } \\
\text { warm-up) }\end{array}$ & WCST-64 & - & $\begin{array}{l}\text { Correct conceptual-level } \\
\text { responses: } \\
\text { moderate-vigorous < rest; } \\
\text { total number of errors: } \\
\text { rest < moderate-vigorous; } \\
\text { perseverative reponses } \\
\text { (total): } \\
\text { rest < moderate-vigorous: } \\
\text { perseverative errors: no } \\
\text { differences }\end{array}$ & No \\
\hline
\end{tabular}

n, number of participants; F, females; "-" = not measured; RT, reaction time; ACC, accuracy; NR, not reported; GAU, Guilford's alternate uses test; CRA, compound remote-association test; WCST, Wisconsin Card Sorting Task; WCST-64, Wisconsin Card Sorting Task (shortened version); $\mathrm{VO}_{2}$ max, maximum volume of oxygen uptake; VO $\mathrm{O}_{2}$ peak, peak oxygen uptake; HRmax, maximum heart rate VT, ventilatory threshold; MET, metabolic equivalent expenditure; PPO, peak power output; Switch Cost = RT difference switching between global to local trials or vice versa.

${ }^{a}$ Exercise duration excludes warm-up time.

${ }^{b}$ Additional sample characteristics provided in Supplementary Table 1. 
TABLE 4 | Summary of studies examining attention during acute aerobic exercise.

\begin{tabular}{|c|c|c|c|c|c|c|c|c|c|}
\hline \multicolumn{3}{|c|}{ Sample characteristics } & \multirow[b]{2}{*}{ Prescribed intensity } & \multicolumn{3}{|c|}{ Exercise } & \multicolumn{2}{|l|}{ Main results } & \multirow[b]{2}{*}{$\begin{array}{l}\text { Did performance } \\
\text { improve? }\end{array}$} \\
\hline Study (year) & $n(F)$ & Age & & $\begin{array}{l}\text { Intensity } \\
\text { category }\end{array}$ & Duration $^{\mathrm{a}}$ & Cognitive task & Response time & Accuracy & \\
\hline $\begin{array}{l}\text { Pesce et al. } \\
\text { (2002) } \\
\text { Experiment } 1\end{array}$ & $16(8)$ & $19-40$ years & Rest, $60 \% \mathrm{VO}_{2} \max$ & Rest, moderate & $N R$ & Local global task & $\begin{array}{l}\text { Overall RT: } \\
\text { moderate < rest; } \\
\text { RT during exercise: } \\
\text { global } \\
\text { targets < local } \\
\text { targets }\end{array}$ & No differences & Yes; only RT \\
\hline $\begin{array}{l}\text { Pesce et al. } \\
\text { (2002) } \\
\text { Experiment } 2\end{array}$ & $\begin{array}{l}\text { Same } \\
\text { participants } \\
\text { and design as } \\
\text { Exp } 1\end{array}$ & & Rest, $60 \% \mathrm{VO}_{2} \max$ & Rest, moderate & NR & Local global task & Moderate $<$ rest & No differences & Yes; only RT \\
\hline $\begin{array}{l}\text { McMorris and } \\
\text { Graydon } \\
\text { (1997) } \\
\text { Experiment } 1\end{array}$ & $\begin{array}{l}12(0) \text { College } \\
\text { soccer players }\end{array}$ & $\begin{array}{c}20.8 \pm 1.34 \\
\text { years }\end{array}$ & Rest, 70, 100\% MAP & $\begin{array}{l}\text { Rest, } \\
\text { moderate, } \\
\text { near-maximal }\end{array}$ & NR & $\begin{array}{l}\text { Visual search in } \\
\text { game simulations }\end{array}$ & $\begin{array}{l}\text { Speed } \\
\text { of search }(\mathrm{RT}) \text { : near- } \\
\text { maximal < moderate, } \\
\text { rest }\end{array}$ & No differences & $\begin{array}{l}\text { Yes; only RT } \\
\text { near-maximal }\end{array}$ \\
\hline $\begin{array}{l}\text { Pesce et al. } \\
\text { (2004) } \\
\text { Experiment } 1\end{array}$ & $42(20)$ & $\begin{array}{l}\text { Males: } \\
21.9 \pm 4.2 \\
\text { Females: } \\
22.5 \pm 4.3 \\
\text { years }\end{array}$ & Rest, 60\% HRR & Rest, vigorous & $\begin{array}{l}\text { NR: 6-8 min } \\
\text { (2 min } \\
\text { warm-up) }\end{array}$ & Local global task & $\begin{array}{l}\text { RT: } \\
\text { Vigorous < Rest }\end{array}$ & No differences & Yes; only RT \\
\hline $\begin{array}{l}\text { Pesce et al. } \\
(2004) \\
\text { Experiment } 2\end{array}$ & $\begin{array}{l}\text { Same } \\
\text { participants } \\
\text { and design as } \\
\text { Exp } 1\end{array}$ & & Rest, 60\% HRR & Rest, vigorous & $\begin{array}{l}\text { NR: 6-8 min } \\
\text { (2 min } \\
\text { warm-up) }\end{array}$ & Local global task & $\begin{array}{l}\text { Overall RT: } \\
\text { Vigorous < rest; RT } \\
\text { during Exercise: } \\
\text { Males < Females }\end{array}$ & No differences & Yes; only RT \\
\hline $\begin{array}{l}\text { Quelhas } \\
\text { Martins et al. } \\
\text { (2013) } \\
\text { Experiment } 1\end{array}$ & $\begin{array}{l}24 \text { (0): Exercise } \\
\text { 12; Control } 12\end{array}$ & $\begin{array}{c}20.50 \pm 0.89 \\
\text { years }\end{array}$ & $\begin{array}{l}\text { Rest, } 60-77 \% \text { HRmax } \\
\text { (ranged from } 60 \text { to } 180 \\
\text { watts across } 4 \text { blocks) }\end{array}$ & $\begin{array}{l}\text { Rest, } \\
\text { moderate- } \\
\text { vigorous }\end{array}$ & $\mathrm{NR}: \sim 8 \mathrm{~min}$ & PASAT & - & $\begin{array}{l}\text { Percentage of } \\
\text { correct responses: } \\
\text { rest }<\text { moderate- } \\
\text { vigorous }\end{array}$ & Yes \\
\hline
\end{tabular}


TABLE 4 | (Continued)

\begin{tabular}{|c|c|c|c|c|c|c|c|c|c|}
\hline \multicolumn{3}{|c|}{ Sample characteristics } & \multirow[b]{2}{*}{ Prescribed intensity } & \multicolumn{3}{|c|}{ Exercise } & \multicolumn{2}{|c|}{ Main results } & \multirow[b]{2}{*}{$\begin{array}{l}\text { Did performance } \\
\text { improve? }\end{array}$} \\
\hline Study (year) & $n(\mathrm{~F})$ & Age & & $\begin{array}{l}\text { Intensity } \\
\text { category }\end{array}$ & Duration $^{\mathrm{a}}$ & Cognitive task & Response time & Accuracy & \\
\hline $\begin{array}{l}\text { González- } \\
\text { Fernández } \\
\text { et al. (2017) } \\
\text { Experiment } 1\end{array}$ & $24(12)$ & $\begin{array}{c}20.29 \pm 0.95 \\
\text { years }\end{array}$ & $\begin{array}{l}40,60,80,100 \% \text { of } \\
\text { VAT }(<50,50-64 \text {, } \\
64-77,85-100 \% \\
\text { HRmax })\end{array}$ & $\begin{array}{l}\text { Very light, light, } \\
\text { moderate, } \\
\text { vigorous- } \\
\text { maximal }\end{array}$ & $\begin{array}{l}5 \text { min (3 min } \\
\text { warm-up) }\end{array}$ & PVT & $\begin{array}{l}\text { RT: very light, light, } \\
\text { moderate }<\text { vigorous- } \\
\text { maximal }\end{array}$ & - & $\begin{array}{l}\text { Yes; only RT at } \\
\text { lower intensity }\end{array}$ \\
\hline $\begin{array}{l}\text { Wohlwend } \\
\text { et al. (2017) }\end{array}$ & $15(15)$ & $\begin{array}{c}24.3 \pm 3.3 \\
\text { years }\end{array}$ & $40,60 \% \mathrm{VO}_{2} \max$ & Light, moderate & $\begin{array}{l}5 \text { min (5 min } \\
\text { warm-up) }\end{array}$ & CPT & No differences & $\begin{array}{l}\text { Accuracy: } \\
\text { moderate < light }\end{array}$ & $\begin{array}{l}\text { No; only ACC at } \\
\text { higher intensity }\end{array}$ \\
\hline $\begin{array}{l}\text { Yagi et al. } \\
\text { (1999) }\end{array}$ & $24(12)$ & $\begin{array}{c}\text { Females: } \\
20.6 \pm 2.5 \\
\text { Males: } \\
19.9 \pm 1.7 \\
\text { years }\end{array}$ & Rest, 130-150 bpm & Rest, moderate & $10 \mathrm{~min}$ & $\begin{array}{l}\text { Visual and auditory } \\
\text { oddball tasks }\end{array}$ & $\begin{array}{l}\text { Overall RT: } \\
\text { moderate < rest: } \\
\text { RT during exercise: } \\
\text { visual } \\
\text { task < auditory } \\
\text { task }\end{array}$ & $\begin{array}{l}\text { Accuracy: } \\
\text { moderate < rest }\end{array}$ & Yes \\
\hline $\begin{array}{l}\text { Ciria et al. } \\
\text { (2019) }\end{array}$ & 20 (0) Cyclists & $M=23.9$ years & $30,80 \% \mathrm{VO}_{2} \max$ & $\begin{array}{l}\text { Very light, } \\
\text { vigorous }\end{array}$ & $\begin{array}{l}20 \mathrm{~min} \text { (10 min } \\
\text { warm-up) }\end{array}$ & Visual oddball task & No differences & No differences & No effects \\
\hline $\begin{array}{l}\text { Sanabria et al. } \\
\text { (2011) }\end{array}$ & $22(2)$ & $\begin{array}{l}18-22 \text { years; } \\
M=22\end{array}$ & $\begin{array}{l}\text { Active rest/very light } \\
\text { (cycling with no } \\
\text { resistance), } 85 \% \\
\text { anaerobic threshold }\end{array}$ & $\begin{array}{l}\text { Very light, } \\
\text { moderate- } \\
\text { vigorous }\end{array}$ & $20 \mathrm{~min}$ & $\begin{array}{l}\text { Posner spatial } \\
\text { cueing task }\end{array}$ & $\begin{array}{l}\text { RT: moderate- } \\
\text { vigorous < very } \\
\text { light }\end{array}$ & - & \\
\hline $\begin{array}{l}\text { Del Giorno } \\
\text { et al. (2010) }\end{array}$ & $31(13)$ & $\begin{array}{c}20.2 \pm 1.1 \\
\text { years }\end{array}$ & Rest, $75 \%$ VT, VT & $\begin{array}{l}\text { Rest, } \\
\text { moderate, } \\
\text { vigorous }\end{array}$ & $\begin{array}{l}25 \text { min (5-min } \\
\text { warm-up) }\end{array}$ & СРТ & - & $\begin{array}{l}\text { False alarms: } \\
\text { rest }<\text { moderate < } \\
\text { vigorous }\end{array}$ & $\begin{array}{l}\text { No; ACC worse at } \\
\text { higher intensity }\end{array}$ \\
\hline $\begin{array}{l}\text { Huertas et al. } \\
\text { (2011) }\end{array}$ & $30(0)$ & $17 \pm 2$ years & $\begin{array}{l}\text { Rest, } 80,90 \% \text { LT ( 75 } \\
\pm 3,86 \pm 3 \% \text { HRmax) }\end{array}$ & $\begin{array}{l}\text { Rest, } \\
\text { moderate, } \\
\text { vigorous }\end{array}$ & $\begin{array}{l}25 \mathrm{~min} \text { (10 min } \\
\text { warm-up) }\end{array}$ & ANT & $\begin{array}{l}\text { Overall RT: } \\
\text { vigorous < rest; } \\
\text { alerting RT: } \\
\text { moderate < rest }\end{array}$ & No differences & Yes; only RT \\
\hline $\begin{array}{l}\text { Hüttermann } \\
\text { and Memmert } \\
\text { (2014) }\end{array}$ & $\begin{array}{l}8 \text { (4) } \\
\text { Non-athletes; } 8 \\
\text { (2) Team sports } \\
\text { athletes }\end{array}$ & $\begin{array}{c}\text { Overall: } \\
25.47 \pm 3.76 \\
\text { years; } \\
\text { Non-athletes: } \\
26.00 \pm 4.27 \\
\text { Athletes: } \\
24.88 \pm 5.72\end{array}$ & $\begin{array}{l}\text { 50, } 60,70 \% \text { HRmax } \\
\text { (performed within } 1 \\
\text { exercise session) }\end{array}$ & $\begin{array}{l}\text { Very light, light, } \\
\text { moderate }\end{array}$ & $\begin{array}{l}30 \text { ( } 5 \text { min } \\
\text { warm-up) }\end{array}$ & $\begin{array}{l}\text { Attentional breadth } \\
\text { task }\end{array}$ & - & $\begin{array}{l}\text { Success rate: } \\
\text { non-athletes across } \\
\text { all exercise } \\
\text { conditions < } \\
\text { athletes; athletes: } \\
\text { very light < light < } \\
\text { moderate; } \\
\text { non-athletes: } \\
\text { moderate < very } \\
\text { light, light }\end{array}$ & $\begin{array}{l}\text { Yes; ACC in } \\
\text { athletes } \\
\text { No; ACC at higher } \\
\text { intensity in } \\
\text { non-athletes }\end{array}$ \\
\hline
\end{tabular}


TABLE 4 | (Continued)

\begin{tabular}{|c|c|c|c|c|c|c|c|c|c|}
\hline \multicolumn{3}{|c|}{ Sample characteristics } & \multirow[b]{2}{*}{ Prescribed intensity } & \multicolumn{3}{|c|}{ Exercise } & \multicolumn{2}{|c|}{ Main results } & \multirow[b]{2}{*}{$\begin{array}{l}\text { Did performance } \\
\text { improve? }\end{array}$} \\
\hline Study (year) & $n(\mathrm{~F})$ & Age & & $\begin{array}{l}\text { Intensity } \\
\text { category }\end{array}$ & Duration $^{\mathrm{a}}$ & Cognitive task & Response time & Accuracy & \\
\hline $\begin{array}{l}\text { Sanchis et al. } \\
\text { (2020) }\end{array}$ & $24(0)$ & $\begin{array}{c}22.6 \pm 2.9 \\
\text { years }\end{array}$ & $80 \%$ VT1, 80\% VT2 & Light, moderate & $\begin{array}{l}33 \mathrm{~min} 45 \mathrm{~s} \\
\text { (6.5 min } \\
\text { warm-up) }\end{array}$ & ANTI-I (Arousal) & $\begin{array}{l}\text { ANT-I overall RT: } \\
\text { moderate < light; } \\
\text { AV RT: no } \\
\text { differences }\end{array}$ & No differences & $\begin{array}{l}\text { Yes; only RT at } \\
\text { moderate }\end{array}$ \\
\hline $\begin{array}{l}\text { Lambourne } \\
\text { et al. (2010) }\end{array}$ & $19(11)$ & $\begin{array}{c}21.37 \pm 0.9 \\
\text { years }\end{array}$ & $\begin{array}{l}\text { Rest, } 90 \% \mathrm{VT} \text { (mean } \\
\mathrm{HR}=143 \pm 13 \mathrm{bpm} \\
\mathrm{RPE}=13 \pm 1)\end{array}$ & Rest, moderate & $\begin{array}{l}35 \mathrm{~min}(5-\mathrm{min} \\
\text { warm-up) }\end{array}$ & PASAT & - & No differences & No effects \\
\hline $\begin{array}{l}\text { Radel et al. } \\
\text { (2018) }\end{array}$ & $\begin{array}{l}12(0) \text { Trained } \\
\text { cyclists }\end{array}$ & $\begin{array}{c}27.8 \pm 2.0 \\
\text { years }\end{array}$ & $\begin{array}{l}\text { Rest, } 50 \text { watts, } \mathrm{VT} \text {, } \\
\mathrm{VT} \pm 15 \% \\
\text { (moderate-varied) }\end{array}$ & $\begin{array}{l}\text { Rest, light, } \\
\text { moderate, } \\
\text { moderate- } \\
\text { varied }\end{array}$ & $\begin{array}{l}40 \mathrm{~min} \text { (10 min } \\
\text { warm-up) }\end{array}$ & SART & $\begin{array}{l}\text { Go RT: moderate, } \\
\text { moderate- } \\
\text { varied < rest, light; } \\
\text { moderate- } \\
\text { varied < moderate }\end{array}$ & $\begin{array}{l}\text { False alarms: } \\
\text { rest < light, } \\
\text { moderate, } \\
\text { moderate-varied }\end{array}$ & $\begin{array}{l}\text { Yes; RT at } \\
\text { moderate No; ACC } \\
\text { at light to moderate }\end{array}$ \\
\hline $\begin{array}{l}\text { González- } \\
\text { Fernández } \\
\text { et al. (2017) } \\
\text { Experiment } 2\end{array}$ & $18(18)$ & $\begin{array}{c}19.94 \pm 1.98 \\
\text { years }\end{array}$ & $\begin{array}{l}\text { Low effort, } 75 \% \text { VAT } \\
\text { ( 44, 63\% HRmax) }\end{array}$ & Very light, light & $\begin{array}{l}45 \text { min } \\
\text { ( } 3 \text { min-warm } \\
\text { up) }\end{array}$ & PVT & Light $<$ very light & - & Yes; only RT at light \\
\hline $\begin{array}{l}\text { Bullock et al. } \\
\text { (2015) }\end{array}$ & $12(6)$ & $\begin{array}{c}20 \pm 1.08 \\
\text { years }\end{array}$ & Rest, 7-9, 12-14 RPE & $\begin{array}{l}\text { Rest, light, } \\
\text { moderate }\end{array}$ & $\begin{array}{l}45 \mathrm{~min}(5-\mathrm{min} \\
\text { warm-up) }\end{array}$ & Visual oddball task & $\begin{array}{l}\text { moderate < rest, } \\
\text { light }\end{array}$ & No differences & $\begin{array}{l}\text { Yes; only RT in } \\
\text { moderate }\end{array}$ \\
\hline
\end{tabular}

Note. n, number of participants; F, females; "-" = not measured; RT, reaction time; ACC, accuracy; NR, not reported; PASAT, Paced Auditory Serial Addition Task; PVT, psychomotor vigilance task; CPT, continuous performance task; ANT, attentional network task; SART, Sustained Attention to Response Task; $V_{2}{ }_{2} m a x$, maximum volume of oxygen uptake; VO 2 peak, peak oxygen uptake; HRmax, maximum heart rate; HRR, hear rate reserve; VT, ventilatory threshold; MAP, maximum aerobic power; VAT, ventilatory anaerobic threshold; RPE, rating of perceived exertion.

${ }^{a}$ Exercise duration excludes warm-up time. 
TABLE 5 | Summary of studies examining motor speed during acute aerobic exercise.

\begin{tabular}{|c|c|c|c|c|c|c|c|c|c|}
\hline \multicolumn{3}{|c|}{ Sample characteristics } & \multirow[b]{2}{*}{ Prescribed intensity } & \multicolumn{3}{|c|}{ Exercise } & \multicolumn{2}{|c|}{ Main results } & \multirow[b]{2}{*}{$\begin{array}{l}\text { Did performance } \\
\text { improve? }\end{array}$} \\
\hline Study (year) & $n(\mathrm{~F})$ & Age & & Intensity category & Duration $^{\mathrm{a}}$ & Cognitive task & Response time & Accuracy & \\
\hline $\begin{array}{l}\text { John et al. } \\
\text { (2009) }\end{array}$ & $20(9)$ & $\begin{array}{l}26.4 \pm 4.04 \\
\text { years }\end{array}$ & Rest, $1 \mathrm{MPH}$ & Rest, very light & NR & $\begin{array}{l}\text { Typing, mouse } \\
\text { proficiency }\end{array}$ & Typing speed: very light $<$ rest & $\begin{array}{l}\text { Mouse proficiency: } \\
\text { moderate < rest }\end{array}$ & Yes \\
\hline $\begin{array}{l}\text { Arcelin et al. } \\
\text { (1998) }\end{array}$ & $22(12)$ & $\begin{array}{c}23.5 \pm 4.3 \\
\text { years }\end{array}$ & Rest, 60\% MAP & Rest, moderate & 310 -min bouts & CRT & Moderate < rest & $\begin{array}{l}\text { Error rate: } \\
\text { moderate < rest }\end{array}$ & Yes \\
\hline $\begin{array}{l}\text { Chmura et al. } \\
\text { (1998) }\end{array}$ & $17(0)$ & $\begin{array}{c}\text { Above LT: } \\
24.8 \pm 1.4 ; \\
\text { Below LT: } \\
22.6 \pm 1.8 \\
\text { years }\end{array}$ & $\begin{array}{l}\text { 70\% Below LT, 10\% } \\
\text { Above LT }\end{array}$ & Very light, moderate & $\begin{array}{l}\text { Above LT: } 20 \mathrm{~min} ; \\
\text { Below LT: } 60 \mathrm{~min}\end{array}$ & CRT & $\begin{array}{l}\text { Min 10-20 very } \\
\text { light < pre-exercise; Min 10-60 } \\
\text { moderate < pre-exercise }\end{array}$ & - & Yes \\
\hline $\begin{array}{l}\text { Ando et al. } \\
\text { (2010) }\end{array}$ & $10(0)$ & $\begin{array}{l}25.1 \pm 3.4 \\
\text { years }\end{array}$ & $\begin{array}{l}\text { Rest, } 40,60,80 \% \\
\mathrm{VO}_{2} \text { peak }\end{array}$ & $\begin{array}{l}\text { Rest, light, moderate, } \\
\text { vigorous }\end{array}$ & $6.5 \mathrm{~min}$ & $\begin{array}{l}\text { SRT to } \\
\text { peripheral } \\
\text { visual stimuli }\end{array}$ & $\begin{array}{l}\text { Overall RT: no differences; } \\
\text { premotor time: rest < vigorous }\end{array}$ & No differences & No, only in vigorous \\
\hline $\begin{array}{l}\text { Ando et al. } \\
\text { (2008) }\end{array}$ & $12(0)$ & $\begin{array}{l}26.2 \pm 3.1 \\
\text { years }\end{array}$ & Rest, $65 \% \mathrm{VO}_{2}$ peak & Rest, vigorous & $10 \mathrm{~min}$ & SRT & $\begin{array}{l}\text { Overall RT: no differences; } \\
\text { peripheral visual premotor time: } \\
\text { rest < vigorous; central visual } \\
\text { premotor time: no differences }\end{array}$ & No differences & No \\
\hline $\begin{array}{l}\text { Arcelin and } \\
\text { Brisswalter } \\
\text { (1999) }\end{array}$ & $19(9)$ & $\begin{array}{l}23.7 \pm 3.3 \\
\text { years }\end{array}$ & Rest, 60\% MAP & Rest, moderate & $10 \mathrm{~min}$ & CRT & No differences & No differences & No \\
\hline $\begin{array}{l}\text { Brisswalter } \\
\text { et al. (1997) }\end{array}$ & $20(0)$ & $\begin{array}{c}\text { Trained: } \\
23.3 \pm 1.5 ; \\
\text { untrained: } \\
23.7 \pm 1.8 \\
\text { years }\end{array}$ & $\begin{array}{l}\text { Rest, } 20,40,60,80 \% \\
\text { MAP }\end{array}$ & $\begin{array}{l}\text { Rest, very light, light, } \\
\text { moderate, vigorous }\end{array}$ & $10 \mathrm{~min}$ & SRT & $\begin{array}{l}\text { RT untrained: light, moderate, } \\
\text { vigorous > rest; RT trained: } \\
\text { light > rest }\end{array}$ & No differences & No \\
\hline $\begin{array}{l}\text { Davranche } \\
\text { and Audiffren } \\
\text { (2004) }\end{array}$ & $16(7)$ & $\begin{array}{l}22.8 \pm 2.5 \\
\text { years }\end{array}$ & Rest, 20, 50\% MAP & $\begin{array}{l}\text { Rest, very light, } \\
\text { moderate }\end{array}$ & $17 \mathrm{~min}$ & CRT & $\begin{array}{l}\text { RT: moderate < rest, very light, } \\
\text { rest }\end{array}$ & No differences & $\begin{array}{l}\text { Yes, only in } \\
\text { moderate }\end{array}$ \\
\hline $\begin{array}{l}\text { Paas and } \\
\text { Adam (1991) }\end{array}$ & $16(4)$ & $\begin{array}{l}26.6 \pm 5.6 \\
\text { years }\end{array}$ & $\begin{array}{l}\text { Rest, } 5,40 / 85,75 \% \\
\text { Wmax }\end{array}$ & $\begin{array}{l}\text { Rest, very light, } \\
\text { moderate-vigorous, } \\
\text { vigorous }\end{array}$ & $\begin{array}{l}20 \min (10 \mathrm{~min} \\
\text { warm-up) }\end{array}$ & CRT & $\begin{array}{l}\text { RT: very light, } \\
\text { moderate-vigorous, } \\
\text { vigorous < rest }\end{array}$ & $\begin{array}{l}\text { Error rate: very } \\
\text { light, } \\
\text { moderate-vigorous, } \\
\text { vigorous < rest }\end{array}$ & Yes \\
\hline $\begin{array}{l}\text { Audiffren et al. } \\
\text { (2008) }\end{array}$ & $17(8)$ & $\begin{array}{c}\text { Women: } \\
21.13 \pm 1.13 \\
\text { Men: } \\
22.00 \pm 1.22 \\
\text { years }\end{array}$ & Rest, 90\% VT & Rest, moderate & $35 \mathrm{~min}$ & CRT & $\begin{array}{l}\text { RT: Min 14-39 moderate }<\text { Min } \\
\text { 14-39 rest }\end{array}$ & - & Yes \\
\hline $\begin{array}{l}\text { Collardeau } \\
\text { et al. (2001) }\end{array}$ & $11(\mathrm{NR})$ & $\begin{array}{l}26.5 \pm 4.8 \\
\text { years }\end{array}$ & Rest, $100 \%$ VT & Rest, vigorous & $90 \mathrm{~min}$ & SRT & Min 40 vigorous < pre-vigorous & No differences & Yes \\
\hline $\begin{array}{l}\text { Collardeau } \\
\text { et al. (2001) }\end{array}$ & $8(N R)$ & $\begin{array}{l}24.3 \pm 3.4 \\
\quad \text { years }\end{array}$ & Rest, $100 \%$ VT & Rest, vigorous & $100 \mathrm{~min}$ & SRT & RT: pre-vigorous < vigorous & No differences & No \\
\hline
\end{tabular}


TABLE 6 | Summary of studies examining information processing during acute aerobic exercise.

\begin{tabular}{|c|c|c|c|c|c|c|c|c|c|}
\hline \multicolumn{3}{|c|}{ Sample characteristics } & \multirow[b]{2}{*}{ Prescribed intensity } & \multicolumn{3}{|c|}{ Exercise } & \multicolumn{2}{|c|}{ Main results } & \multirow[b]{2}{*}{$\begin{array}{l}\text { Did performance } \\
\text { improve? }\end{array}$} \\
\hline Study (year) & $n(\mathrm{~F})$ & Age & & Intensity category & Duration $^{a}$ & Cognitive task & Response time & Accuracy & \\
\hline $\begin{array}{l}\text { McMorris and } \\
\text { Graydon } \\
\text { (1997) } \\
\text { Experiment } 1\end{array}$ & $12(0)$ & $\begin{array}{c}20.8 \pm 1.34 \\
\text { years }\end{array}$ & Rest, $70,100 \%$ MAP & $\begin{array}{l}\text { Rest, vigorous, } \\
\text { near-maximal }\end{array}$ & $N R$ & $\begin{array}{l}\text { Visual search in } \\
\text { game } \\
\text { simulations }\end{array}$ & Near-maximal < vigorous, rest & - & $\begin{array}{l}\text { Yes; only RT in } \\
\text { near-max }\end{array}$ \\
\hline $\begin{array}{l}\text { McMorris and } \\
\text { Graydon } \\
\text { (1997) } \\
\text { Experiment } 2\end{array}$ & $12(0)$ & $\begin{array}{l}20.8 \pm 1.78 \\
\text { years }\end{array}$ & Rest, 70, 100\% MAP & $\begin{array}{l}\text { Rest, vigorous, } \\
\text { near-maximal }\end{array}$ & $N R$ & $\begin{array}{l}\text { Soccer } \\
\text { decision- } \\
\text { making/problem } \\
\text { solving }\end{array}$ & $\begin{array}{l}\text { Total speed of decision: } \\
\text { vigorous, near-maximal < rest; } \\
\text { speed of decision following ball } \\
\text { detection: near-maximal < rest, } \\
\text { vigorous }\end{array}$ & $\begin{array}{l}\text { Accuracy: } \\
\text { rest }<\text { near- } \\
\text { maximal }\end{array}$ & Yes \\
\hline $\begin{array}{l}\text { Adam et al. } \\
\text { (1997) }\end{array}$ & $20(9)$ & $\begin{array}{l}26.4 \pm 5.1 \\
\text { years }\end{array}$ & $5,75 \%$ Wmax & Very light, vigorous & $\begin{array}{l}20 \min (10 \mathrm{~min} \\
\text { warm-up) }\end{array}$ & $\begin{array}{l}\text { SIT decision } \\
\text { task; STM } \\
\text { decision task }\end{array}$ & Vigorous < very light & No differences & $\begin{array}{l}\text { Yes; only RT in } \\
\text { higher intensity }\end{array}$ \\
\hline $\begin{array}{l}\text { Paas and } \\
\text { Adam (1991) }\end{array}$ & $16(4)$ & $\begin{array}{c}26.6 \pm 5.6 \\
\text { years }\end{array}$ & $\begin{array}{l}\text { Rest, } 5,40 / 85,75 \% \\
\text { Wmax }\end{array}$ & $\begin{array}{l}\text { Rest, very light, } \\
\text { moderate-vigorous, } \\
\text { vigorous }\end{array}$ & $\begin{array}{l}20 \min (10 \mathrm{~min} \\
\text { warm-up) }\end{array}$ & $\begin{array}{l}\text { Backward } \\
\text { masking task }\end{array}$ & - & $\begin{array}{l}\text { Letters correct: } \\
\text { during } \\
\text { exercise < before, } \\
\text { after exercise }\end{array}$ & No \\
\hline $\begin{array}{l}\text { Shields et al. } \\
\text { (2011) }\end{array}$ & $33(17)$ & $\begin{array}{l}\text { Women: } \\
20.7 \pm 1.9 \\
\text { Men: } \\
23.1 \pm 3.5 \\
\quad \text { years }\end{array}$ & Rest, 45, 80\% HRmax & $\begin{array}{l}\text { Rest, very light, } \\
\text { vigorous }\end{array}$ & $20 \mathrm{~min}$ & $\begin{array}{l}\text { Visual threat } \\
\text { detection }\end{array}$ & Very light, vigorous < rest & $\begin{array}{l}\text { Overall accuracy: } \\
\text { rest < very light, } \\
\text { vigorous; } \\
\text { discrepant } \\
\text { fear-irrelevant } \\
\text { accuracy: } \\
\text { vigorous < very } \\
\text { light }\end{array}$ & Yes \\
\hline $\begin{array}{l}\text { Lambourne } \\
\text { et al. (2010) }\end{array}$ & $19(11)$ & $\begin{array}{l}21.1 \pm 1.7 \\
\text { years }\end{array}$ & $\begin{array}{l}\text { Rest, } 90 \% \mathrm{VT} \text { (mean } \\
\mathrm{HR}=143 \pm 13 \mathrm{bpm} \\
\mathrm{RPE}=13 \pm 1)\end{array}$ & Rest, moderate & $40 \mathrm{~min}$ & CFF & - & $\begin{array}{l}\text { CFF score: Min 28, } \\
\text { Min } 30 \text { rest < Min } \\
28, \text { Min } 30 \\
\text { moderate }\end{array}$ & Yes \\
\hline $\begin{array}{l}\text { Grego et al. } \\
(2004)\end{array}$ & $16(0)$ & $\begin{array}{l}\text { Endurance- } \\
\text { trained: } \\
30.8 \pm 7.3 \\
\text { regular trained: } \\
29.4 \pm 4.8 \\
\text { years }\end{array}$ & Rest, $60 \% \mathrm{VO}_{2} \max$ & Rest, moderate & $180 \mathrm{~min}$ & CFF & - & $\begin{array}{l}\text { CFF mdi: Min } 120 \\
\text { moderate < Min } 20 \\
\text { moderate; CFF } \\
\text { mtot: no differences }\end{array}$ & No \\
\hline
\end{tabular}


TABLE 7 | Summary of studies examining memory during acute aerobic exercise.

\begin{tabular}{|c|c|c|c|c|c|c|c|c|c|c|c|}
\hline \multicolumn{3}{|c|}{ Sample characteristics } & \multirow[b]{2}{*}{$\begin{array}{l}\text { Prescribed } \\
\text { intensity }\end{array}$} & \multicolumn{2}{|c|}{ Exercise } & \multicolumn{3}{|c|}{ Stage of memory } & \multicolumn{2}{|r|}{ Main results } & \multirow[b]{2}{*}{$\begin{array}{l}\text { Did performance } \\
\text { improve? }\end{array}$} \\
\hline Study (year) & $n(F)$ & Age & & $\begin{array}{l}\text { Intensity } \\
\text { category }\end{array}$ & Duration ${ }^{\mathrm{a}}$ & Start encoding ${ }^{\mathrm{b}}$ & Start retrieval & Cognitive task & Response time & Accuracy & \\
\hline $\begin{array}{l}\text { Pyke et al. } \\
\text { (2020) } \\
\text { Experiment } 3\end{array}$ & $23(20)$ & $\begin{array}{l}19.62 \pm 1.51 \\
\text { years }\end{array}$ & $\begin{array}{l}\text { High-intensity } \\
\text { interval training } \\
\text { (HIIT), 65-75\% } \\
\text { HRmax }\end{array}$ & $\begin{array}{l}\text { Moderate, } \\
\text { vigorous }\end{array}$ & $6 \mathrm{~min}$ & Immediately pre-exercise & $\begin{array}{l}90 \text { min } \\
\text { post-encoding }\end{array}$ & $\begin{array}{l}\text { Old/New } \\
\text { recognition task }\end{array}$ & & Moderate $>$ passive rest & Yes \\
\hline $\begin{array}{l}\text { Keyan and } \\
\text { Bryant (2017b) }\end{array}$ & $49(33)$ & $\begin{array}{l}\text { Exercise: } \\
19.96 \pm 2.32 \\
\text { Walking: } 19.79 \\
\pm 2.70 \text { years }\end{array}$ & $\begin{array}{l}\text { Walking, } 50-85 \% \\
\text { HRmax }\end{array}$ & $\begin{array}{l}\text { Very } \\
\text { light-vigorous }\end{array}$ & $10 \mathrm{~min}$ & Immediately pre-exercise & $\begin{array}{l}2 \text { days } \\
\text { post-exercise }\end{array}$ & Cued recall & & $\begin{array}{l}\text { Cued recall: no differences; Intrusive } \\
\text { Memories: walk < exercise }\end{array}$ & Yes \\
\hline $\begin{array}{l}\text { Miles and } \\
\text { Hardman } \\
\text { (1998) }\end{array}$ & $24(18)$ & $M=20.3$ years & $120-150 \mathrm{bpm}$ & $\begin{array}{l}\text { Very } \\
\text { light-moderate }\end{array}$ & $11 \mathrm{~min}$ & During exercise, rest & $\begin{array}{l}\text { During } \\
\text { exercise, rest }\end{array}$ & Word list recall & - & $\begin{array}{l}\text { Correct Free recall: exercise-rest, } \\
\text { rest-exercise < rest-rest, } \\
\text { exercise-exercise; false alarms: no } \\
\text { differences }\end{array}$ & No effects \\
\hline $\begin{array}{l}\text { Crawford et al. } \\
\text { (2021) } \\
\text { Experiment } 2\end{array}$ & $68(38)$ & $\begin{array}{l}20.79 \pm 1.98 \\
\text { years }\end{array}$ & $80 \% \mathrm{HRR}$ & Vigorous & $15 \mathrm{~min}$ & $\begin{array}{l}\text { Immediately pre-exercise } \\
\text { (List 1), } 5 \text { min after (List } \\
\text { 2) }\end{array}$ & $\begin{array}{l}\text { Immediately } \\
\text { pre-exercise } \\
\text { (list 1), } 5 \text { min } \\
\text { after (List 2) }\end{array}$ & $\begin{array}{l}\mathrm{AB} / \mathrm{AC} \text { memory } \\
\text { interference task }\end{array}$ & & $\begin{array}{l}\text { Memory interference: } \\
\text { vigorous > rest }\end{array}$ & No \\
\hline $\begin{array}{l}\text { Frith et al. } \\
(2017)\end{array}$ & $88(48)$ & $\begin{array}{l}21.9 \pm 2.4 \\
\text { years }\end{array}$ & Rest, self-selected & $\begin{array}{l}\text { Rest, light to } \\
\text { near-maximal }\end{array}$ & $15 \mathrm{~min}$ & $\begin{array}{l}\text { Immediately before, min } \\
\text { NR during, min } 5 \text { AFTER }\end{array}$ & $\begin{array}{l}\text { Min } 20, \mathrm{Hr} 24 \\
\text { post-exercise }\end{array}$ & $\begin{array}{l}\text { RAVLT; prospective } \\
\text { memory task }\end{array}$ & & $\begin{array}{l}\text { Short-term memory: no differences; } \\
\text { learning: no differences; } 20 \text {-min } \\
\text { long-term memory: exercise during } \\
\text { encoding < exercise pre-encoding, rest; } \\
\text { 24-h long-term memory: no differences; } \\
\text { 24-h attribution memory: exercise during } \\
\text { encoding < exercise pre-encoding; } \\
\text { prospective memory: no differences }\end{array}$ & Yes \\
\hline $\begin{array}{l}\text { Loprinzi et al. } \\
\text { (2021) }\end{array}$ & $150(88)$ & $\begin{array}{l}\text { Exercise: } \\
20.32 \pm 1.3 \\
\text { Control: } 20.17 \\
\pm 1.3 \text { years }\end{array}$ & $80 \% \mathrm{HRR}$ & Vigorous & $20 \mathrm{~min}$ & $\begin{array}{l}\text { Incidental encoding } \\
\text { immediately } \\
\text { pre-exercise, intentional } \\
\text { encoding immediately } \\
\text { post-exercise }\end{array}$ & $\begin{array}{l}0,30 \mathrm{~min} \\
\text { post-exercise }\end{array}$ & $\begin{array}{l}\text { Incidental memory } \\
\text { processing task, } \\
\text { incidental encoding } \\
\text { task }\end{array}$ & - & No differences & No effects \\
\hline $\begin{array}{l}\text { Loprinzi et al. } \\
\text { (2021) } \\
\text { Experiment } 1\end{array}$ & $47(27)$ & $\begin{array}{l}21.1 . \pm 1.7 \\
\text { years }\end{array}$ & $75 \% \mathrm{HRR}$ & Vigorous & $20 \mathrm{~min}$ & 5 min post-exercise & $\begin{array}{l}55 \text { min, } 24 \mathrm{~h} \\
\text { post-encoding }\end{array}$ & Word list task & - & $\begin{array}{l}\text { Exercise > rest through encoding, } \\
\text { retrieval }\end{array}$ & Yes \\
\hline $\begin{array}{l}\text { Loprinzi et al. } \\
\text { (2021) } \\
\text { Experiment } 2\end{array}$ & $42(23)$ & $\begin{array}{l}20.6 \pm 1.1 \\
\text { years }\end{array}$ & $75 \%$ HRR & Vigorous & $20 \mathrm{~min}$ & Immediately pre-exercise & $\begin{array}{l}4 \mathrm{~h}, 20 \mathrm{~min}, 24 \\
\mathrm{~h} \\
\text { post-encoding }\end{array}$ & Word list task & & Exercise $>$ rest through consolidation & Yes \\
\hline $\begin{array}{l}\text { Loprinzi et al. } \\
(2021) \\
\text { Experiment } 3\end{array}$ & $31(27)$ & $\begin{array}{l}20.5 \pm 1.0 \\
\text { years }\end{array}$ & $75 \% \mathrm{HRR}$ & Vigorous & $20 \mathrm{~min}$ & $2 \mathrm{~h}$ pre-exercise & $\begin{array}{l}4 \mathrm{~h}, 24 \mathrm{~h} \\
\text { post-encoding }\end{array}$ & Word list task & & No differences & No effects \\
\hline $\begin{array}{l}\text { Silvers et al. } \\
\text { (2018) }\end{array}$ & $72(49)$ & $\begin{array}{l}20.6 \pm 1.9 \\
\text { years }\end{array}$ & $\begin{array}{l}\text { Rest, } 40,60,80 \% \\
\text { HRmax }\end{array}$ & $\begin{array}{l}\text { Rest, very } \\
\text { light, light, } \\
\text { vigorous }\end{array}$ & $20 \mathrm{~min}$ & During exercise, rest & $\begin{array}{l}\text { Immediately, } 1 \\
\text { Week } \\
\text { Post-exercise }\end{array}$ & $\begin{array}{l}\text { Multiple choice } \\
\text { recall }\end{array}$ & - & No differences & No effects \\
\hline
\end{tabular}


TABLE 7 | Summary of studies examining memory during acute aerobic exercise.

\begin{tabular}{|c|c|c|c|c|c|c|c|c|c|c|c|}
\hline \multicolumn{3}{|c|}{ Sample characteristics } & \multirow[b]{2}{*}{$\begin{array}{l}\text { Prescribed } \\
\text { intensity }\end{array}$} & \multicolumn{2}{|c|}{ Exercise } & \multicolumn{3}{|c|}{ Stage of memory } & \multicolumn{2}{|r|}{ Main results } & \multirow[b]{2}{*}{$\begin{array}{l}\text { Did performance } \\
\text { improve? }\end{array}$} \\
\hline Study (year) & $n(\mathrm{~F})$ & Age & & $\begin{array}{l}\text { Intensity } \\
\text { category }\end{array}$ & Durationa $^{a}$ & Start encoding ${ }^{b}$ & Start retrieval & Cognitive task & Response time & Accuracy & \\
\hline $\begin{array}{l}\text { Keyan and } \\
\text { Bryant (2017a) }\end{array}$ & $54(26)$ & $\begin{array}{l}19.48 \pm 3.03 \\
\text { years }\end{array}$ & $\begin{array}{l}60-70 \mathrm{rpm}(76 \% \\
\text { MAP) }\end{array}$ & Vigorous & $\sim 25 \mathrm{~min}$ & $\begin{array}{l}2 \text { days pre-exercise } \\
\text { [reactivity condition } \\
\text { during exercise] }\end{array}$ & $\begin{array}{l}2 \text { days } \\
\text { post-exercise }\end{array}$ & Cued recall & - & $\begin{array}{l}\text { Recall for central details: reactivation } \\
\text { alone, vigorous alone < reactivation + } \\
\text { vigorous; recall for peripheral details: no } \\
\text { differences; intrusive memories: no } \\
\text { differences }\end{array}$ & Yes \\
\hline $\begin{array}{l}\text { Hötting et al., } \\
2016\end{array}$ & $81(40)$ & $\begin{array}{l}22.00 \pm 2.36 \\
\text { years }\end{array}$ & $\begin{array}{l}\text { Rest, } 57,80 \% \\
\text { HRmax }\end{array}$ & $\begin{array}{l}\text { Rest, very } \\
\text { light-light, } \\
\text { vigorous }\end{array}$ & $30 \mathrm{~min}$ & 10-min pre-exercise & $\begin{array}{l}\text { 20-min, 24-h } \\
\text { post-exercise }\end{array}$ & Vocabulary test & & $\begin{array}{l}\text { Memory 20-min post-exercise (60-min } \\
\text { post-encoding): No differences; memory } \\
\text { 24-h post-exercise: rest < v vigorous }\end{array}$ & Yes \\
\hline $\begin{array}{l}\text { Labban and } \\
\text { Etnier (2011) }\end{array}$ & $48(33)$ & $\begin{array}{l}M=22.02 \\
\text { years }\end{array}$ & RPE 13-15 & $\begin{array}{l}\text { Moderate- } \\
\text { vigorous }\end{array}$ & $30 \mathrm{~min}$ & $\begin{array}{l}\text { Immediately } \\
\text { pre-exercise, } \\
\text { immediately } \\
\text { post-exercise }\end{array}$ & $\begin{array}{l}\text { 35-min } \\
\text { post-encoding }\end{array}$ & $\begin{array}{l}\text { New York University } \\
\text { paragraph recall } \\
\text { test }\end{array}$ & - & $\begin{array}{l}\text { Recall: encoding at rest < encoding } \\
\text { post-exercise, encoding pre-exercise }\end{array}$ & Yes \\
\hline $\begin{array}{l}\text { Pyke et al. } \\
\text { (2020) } \\
\text { Experiment } 1\end{array}$ & 19 (11) & $\begin{array}{l}21.85 \pm 2.43 \\
\text { years }\end{array}$ & $\begin{array}{l}\text { 55-65, 65-75, } \\
75-85 \% \text { HRmax }\end{array}$ & $\begin{array}{l}\text { Light, } \\
\text { moderate, } \\
\text { vigorous }\end{array}$ & $30 \mathrm{~min}$ & Immediately pre-exercise & $\begin{array}{l}80 \text { min } \\
\text { post-encoding }\end{array}$ & $\begin{array}{l}\mathrm{Old} / \mathrm{New} \\
\text { recognition task }\end{array}$ & No differences & Moderate > vigorous & Yes \\
\hline $\begin{array}{l}\text { Wang et al. } \\
(2020)\end{array}$ & $22(0)$ & $\begin{array}{l}21.6 \pm 3.0 \\
\text { years }\end{array}$ & $60-70 \%$ HRmax & Moderate & $30 \mathrm{~min}$ & $\begin{array}{l}\text { Immediately } \\
\text { pre-exercise, } \\
\text { immediately } \\
\text { post-exercise }\end{array}$ & $\begin{array}{l}1,24 \mathrm{~h} \\
\text { post-encoding }\end{array}$ & $\begin{array}{l}\text { DM Task, SRTT, } \\
\text { Procedural memory }\end{array}$ & $\begin{array}{l}\text { SRTTRT } \\
\text { Post-acquisition } \\
\text { exercise < control }\end{array}$ & $\begin{array}{l}\text { Words recalled: exercise pre-acquisition } \\
\text { > post-acquisition, control at } 1 \mathrm{~h} \text {, } \\
\text { pre-acquisition > control at } 24 \mathrm{~h} \text {; word } \\
\text { recognition: exercise pre-acquisition, } \\
\text { post-acquisition > control }\end{array}$ & Yes \\
\hline $\begin{array}{l}\text { van Dongen } \\
\text { et al. (2016) }\end{array}$ & $72(48)$ & $\begin{array}{l}\text { No exercise: } \\
22.6 \pm 2.8 ; \\
\text { immediate } \\
\text { exercise: } 21.5 \\
\pm 2.1 ; \text { delayed } \\
\text { exercise: } 21.6 \\
\pm 2.4 \text { years }\end{array}$ & $80 \%$ HRmax & Vigorous & $35 \mathrm{~min}$ & $\begin{array}{l}\text { Immediately pre-exercise } \\
\text { [Immediate], } 4 \mathrm{~h} \\
\text { pre-exercise [Delayed] }\end{array}$ & $\begin{array}{l}2 \text { days } \\
\text { post-exercise }\end{array}$ & Cued recall & - & $\begin{array}{l}\text { Recall: immediate exercise, no exercise } \\
<\text { delayed exercise }\end{array}$ & Yes \\
\hline $\begin{array}{l}\text { Grego et al. } \\
(2004)\end{array}$ & $16(0)$ & $\begin{array}{l}\text { Endurance- } \\
\text { trained: } 30.8 \pm \\
7.3 \text {; regular } \\
\text { trained: } 29.4 \pm \\
4.8 \text { years }\end{array}$ & $60 \% \mathrm{VO}_{2} \max$ & Moderate & $180 \mathrm{~min}$ & $\begin{array}{l}\text { Min 20, } 40,60,80,100, \\
120,140,160,180 \\
\text { During, Min } 5 \text { After }\end{array}$ & $\begin{array}{l}\text { Min 20, 40, } \\
60,80,100 \\
\text { 120, } 140,160, \\
180 \text { During, } \\
\text { Min } 5 \text { After }\end{array}$ & Map recognition & $\begin{array}{l}\text { Speed recognition: } \\
80,100,120 \mathrm{~min}< \\
20 \mathrm{~min} \text {; errors: } 60, \\
80,100 \mathrm{~min}<20 \\
\text { min }\end{array}$ & Errors: Min 60, 80, $100<\operatorname{Min} 20$ & Yes \\
\hline
\end{tabular}

Note. n, number of participants; F, females; "-", not measured; RT, reaction time; AC, accuracy; NR, not reported; RAVLT, Rey Auditory Verbal Learning Test; DM, Declarative Memory Task; SRTT, Serial Reaction Time Task; $V_{2} \mathrm{O}_{2} \mathrm{max}$, maximum volume of oxygen uptake; $\mathrm{VO}_{2}$ peak, peak oxygen uptake; HRmax, maximum heart rate; HRR, heart rate reserve; VT, ventilatory threshold; PPO, peak power output; MAP = maximum aerobic power; VA, ventilatory anaerobic threshold; RPE, rating of perceived exertion.

${ }^{a}$ Exercise duration excludes warm-up time.

${ }^{b}$ Start of encoding must have taken place before or during exercise to be included. 
TABLE 8 | Classification of aerobic exercise intensity.

Relative intensity

\begin{tabular}{|c|c|c|c|c|}
\hline \multirow[b]{2}{*}{ Intensity } & \\
\hline & $\% \mathrm{VO}_{2} \max$ & \%HRmax & $\% \mathrm{HRR}$ or $\% \mathrm{VO}_{2} \mathrm{R}$ & Perceived exertion (rating on 6-20 RPE scale) \\
\hline Very light & $<37$ & $<57$ & $<30$ & RPE $<9$ (“Very light”) \\
\hline Light & $37-45$ & $57-63$ & $30-39$ & RPE 9-11 ("Very light to fairly light") \\
\hline Moderate & $45-63$ & $64-76$ & $40-59$ & RPE 12-13 ("Fairly light to somewhat hard") \\
\hline Vigorous & $64-90$ & $77-95$ & $60-89$ & RPE 14-17 ("Somewhat hard to very hard") \\
\hline Near-maximal to maximal & $\geq 91$ & $\geq 96$ & $\geq 90$ & RPE $\geq 18$ ("Very hard to maximal exertion") \\
\hline
\end{tabular}

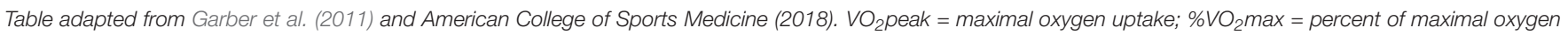
uptake; HRmax, maximal HR; = \%HRmax = percent of maximal HR; HRR, HR reserve; $\mathrm{VO}_{2} R$, oxygen uptake reserve; RPE, ratings of perceived exertion (Borg, 1982).

more research is needed since to date there are very few studies investigating these lower levels of exercise intensity.

Moderate intensity exercise consistently improves response time on inhibition tasks. In total, twelve studies have investigated motor response inhibition or interference control while participants either ran or cycled at moderate intensity, compared to a rest condition. Improvements in inhibitory control are primarily reported as improvements in response times across seven studies, with one study also demonstrating improved accuracy (Davranche et al., 2009; Ando et al., 2014; Komiyama et al., 2015, 2016, 2017; Olson et al., 2016; Finkenzeller et al., 2019). Three studies found decrements in inhibitory control at moderate intensity. Specifically, two found reductions in accuracy and one found slower response time when stimuli and responses are incompatible (Davranche and McMorris, 2009; Olson et al., 2016; Finkenzeller et al., 2019). In contrast, four studies reported no differences in either response times or accuracy, compared to rest (McMorris et al., 2009; Ando et al., 2011; Huertas et al., 2011; Komiyama et al., 2019). In sum, regardless of task type, improved response time is the most consistently observed finding impacting inhibition during moderate intensity when compared to rest. This is consistent with previous meta-analytic investigations demonstrating differing effects of acute exercise on response time vs. accuracy (McMorris and Hale, 2012).

Diving deeper, we find that a more consistent pattern emerges for tasks measuring motor response inhibition compared to those measuring aspects of interference control during moderate exercise. Specifically, relative to rest, response time improves on the Go-No/Go task during exercise, with no reported decrements in either response time or accuracy of performance (Ando et al., 2014; Komiyama et al., 2015, 2016, 2017, 2019). When compared to rest, accuracy does not appear to be significantly altered on less cognitively challenging non-selective stopping tasks, such as the GoNo/Go.

In contrast, when examining interference control at moderate intensity, results are more heterogeneous. Relative to rest, two studies reported overall improvements in response time regardless of stimulus-response compatibility (congruent or incongruent) (Davranche and McMorris, 2009; Davranche et al., 2009), whereas one reported improved speed for congruent trials and another reported slowed response times when switching from incongruent to congruent trials (Davranche and McMorris, 2009; Finkenzeller et al., 2019). Taken together, it appears that response time decreases to a similar extent on interference control and motor response inhibition tasks during acute moderate exercise. With regards to accuracy on interference tasks, one study found greater incongruent errors during acute moderate exercise (Olson et al., 2016), but negligible effects on response times or accuracy have also been reported (Ando et al., 2011; Huertas et al., 2011). In sum, decrements to performance on interference control tasks during moderate intensity stem from either slowed responding or reduced accuracy, specifically on incongruent trials. In contrast, ability to respond appropriately on congruent trials requiring less cognitive control appears to be unaltered. However, given that reported accuracy scores are often at ceiling (reported accuracy rates $>88 \%$ ), the mixed findings with regards to accuracy of inhibitory control at moderate intensity may be due to failure to choose tasks which are complex enough to detect acute exercise-induced changes (McMorris and Hale, 2012).

Vigorous intensity has differing effects on response time and accuracy. Six studies investigated performance under vigorous intensity exercise compared to rest. Two studies demonstrated improved response times on Go/No-Go (Ando et al., 2013) or ANT tasks (Huertas et al., 2011 [marginal significance: $p<0.063]$ ). In contrast, three studies demonstrated reductions in accuracy with no subsequent changes to response time (Ando et al., 2011; Schmit et al., 2015; Komiyama et al., 2019) and one study demonstrated decrements to both response time and accuracy (McMorris et al., 2009). Interestingly, similar to the decrements in performance reported at moderate intensity, these reductions in accuracy are specifically driven by No-Go or incongruent errors, where a participant fails to inhibit a prepotent response and/or responds to task-irrelevant aspects of the stimuli. No decrements were observed for congruent trials, suggesting that task conditions that elicit a higher level of conflict and require greater cognitive control may be more selectively influenced by acute exercise, and more so lead to impairments under higher levels of physical exertion. However, lack of differences in response time or accuracy between vigorous intensity and rest conditions have also been reported (Smith et al., 2016).

Increasing intensity to near-maximal may result in decrements to both response time and accuracy. To date, one study has demonstrated increased response times and reduced accuracy during maximal compared to both vigorous intensity and rest conditions (Smith et al., 2016). Interestingly, 
Schmit et al. (2015) observed a similar trend toward increased incongruent errors in the terminal period before exhaustion, suggesting that physiologically demanding conditions may impair multiple aspects of inhibitory control. However, further work is needed exploring cognitive performance under maximal effort before results can be generalized.

\section{Working Memory}

Very light and light intensities exert variable effects on working memory (see Table 2). For example, Quelhas Martins et al. (2013) found that response latency slopes were lower on a Sternberg task, indicating faster response times during light intensity cycling, whereas no differences were found during very light intensity, compared to rest. No effects on response times or accuracy between light, moderate and vigorous intensity conditions have also been reported (Travlos and Marisi, 1995).

Increasing intensity from very light-to-light to moderate may result in slight improvements or no changes in working memory. For example, compared to rest, two studies found improvements in response times, with no improvements in accuracy on Sternberg and speed match tasks (Quelhas Martins et al., 2013; Rattray and Smee, 2016). Furthermore, Rattray and Smee (2016) found that improved response times were demonstrated during moderate intensity conditions under both a constant or varied load. When measured using a spatial delayed response task, three studies found no differences in working memory during moderate vs. rest (Komiyama et al., 2016, 2017, 2019). Thus, across a variety of tasks, improvements or null effects have been reported.

Finally, of the three studies to assess vigorous intensity exercise, two found impairments in accuracy with no changes in response time compared to rest (Komiyama et al., 2019) and at the end vs. beginning of heavy exercise (Tempest et al., 2017), and one found no differences between light, moderate and vigorous intensities (Travlos and Marisi, 1995).

\section{Cognitive Flexibility}

Very light-to-light intensities may impair certain aspects of cognitive flexibility and enhance others (see Table 3). Very light intensity exercise differentially affects convergent and divergent thinking. For instance, in two experiments, Oppezzo and Schwartz (2014) measured performance on the GAU and CRA tasks during a self-selected "comfortable" walking condition, characterized as very-light to light intensity. Results demonstrated convergent thinking was impaired, with the number of correct responses generated during the CRA decreasing during exercise compared to rest (Experiment 2), whereas the number of creative, appropriate alternative uses generated during GAU increased (Experiments 1 and 2). Thus, convergent thinking may suffer, while divergent thinking may improve during bouts of light intensity exercise. However, intensity was not specifically determined using ACSM criteria and fitness level of participants and exercise duration were not reported, thus results should be interpreted with caution. More research is needed to understand how convergent and divergent thinking may be affected under varying exercise intensities. Additionally, more work is needed to understand how perseveration and set-shifting are affected during light intensity exercise. To date, two studies examined performance, finding an increase in the number of unique errors on the WCST during exercise compared to rest, or no differences between light intensity and rest (Del Giorno et al., 2010; Wang et al., 2013). Thus, light intensity may impair or enhance cognitive flexibility depending on the task used.

At moderate exercise intensity, cognitive flexibility remains relatively unaffected. For example, Pesce et al. (2003) found quicker response times when switching between local and global attending, with no changes in accuracy during exercise vs. rest. Labelle et al. (2013) found no differences between moderate and light intensity conditions on a modified Stroop task with a switching condition. Finally, Wang et al. (2013) found no differences in performance on the WCST between moderate, light and rest conditions.

At vigorous intensities, response times may be improved for certain aspects of cognitive flexibility, whereas accuracy may be impaired for others. For example, three studies assessed cognitive flexibility using the WSCT. Results and specific dependent outcomes variables varied between studies, but overall, performance was impaired during vigorous intensity exercise, compared to lower intensities or rest. When examined between-subjects, Dietrich and Sparling (2004) found an increase in the number of conceptual level responses and an increase in total number of errors when participants ran or cycled at an intensity ranging from moderate-to-vigorous (i.e., 70-80\% HRmax), compared to rest. Similarly, Wang et al. (2013) found that participants made more perseverative errors, completed fewer categories and made fewer conceptual level responses under vigorous intensities compared to moderate, light and rest conditions. When examined within-subjects, performance also declined under vigorous exercise intensities compared to rest (Del Giorno et al., 2010). In contrast, the ability to switch between local and global processing may be enhanced. One study demonstrated overall improvements in response times, as well as quicker speed when switching between global to local trials, or vice versa, during vigorous intensities compared to rest (Pesce et al., 2007). Thus, similar to light intensity, vigorous intensity may impair certain aspects of cognitive flexibility and enhance others but effects are often dependent on the cognitive task used.

\section{Integrative Summary of Intensity Effects on Executive Functions}

Prior meta-analytic reviews have demonstrated that executive functions show beneficial and significantly larger effects than any other category of cognitive tasks (e.g., information processing, simple and choice reaction time, attention, memory) (Lambourne and Tomporowski, 2010; Chang et al., 2012). However, these effects were not dependent on exercise intensity, contradicting theoretical predictions where higher levels intensity are thought to hinder these higher-order cognitive processes (Yerkes and Dodson, 1908; Dietrich, 2003; McMorris et al., 2008; Dietrich and Audiffren, 2011). Notably, empirical acceptance that higher exercise intensities induce lower executive performance than moderate intensities has been mixed. However, conclusions 
drawn here and previously regarding executive function during exercise rely heavily on studies examining inhibitory control. Examining executive functions separately may lead to an ability to detect domain-specific intensity effects during exercise.

Here we find intensity-dependent effects for inhibition, such that moderate exercise intensity improves response time and moderate-to-vigorous intensity impairs accuracy. This pattern of results aligns with previous reviews, suggesting that complex cognitive tasks are more likely to be affected by exercise than simple tasks (McMorris and Graydon, 2000; Dietrich, 2006; Chang et al., 2012). This deterioration in inhibition under higher exercise intensities provides support for hypofrontality temporarily impairing executive function (Dietrich and Audiffren, 2011; Audiffren, 2016). Further, the declines in performance demonstrated during the initial minutes of exercise, or during heightened levels of physical load (as induced by intensity) may be the result of competing physiological resources. This competition may lead to reduced ability to inhibit prepotent motor responses and selectively attend and respond to target stimuli whilst ignoring goal-irrelevant stimuli. However, heterogeneity still exists and performance is often dependent on several moderating factors, including duration or fitness level.

In contrast, the impact of exercise intensity is less clear for working memory and cognitive flexibility. To assume these follow similar patterns demonstrated for inhibition with regards to effects of exercise intensity on response time and accuracy may be premature. For instance, here we find no clear systematic effects of exercise intensity on working memory performance, instead performance consistently declines across light, moderate and vigorous intensities. This is in contrast with inverted$U$ and hypofrontality hypotheses, but it aligns with previous work reporting a detrimental effect of moderate intensity on working memory tasks (McMorris et al., 2011). Different from working memory, cognitive flexibility shows clear intensitydependent effects for accuracy and no consistent effects on response time. However, these effects appear to be moderated by the particular type of task used to assess performance (see Table 7). Furthermore, types of study designs used to measure working memory were more variable than cognitive flexibility and inhibition, which predominantly compared intensities of interest to a rest condition. Taken together, the findings are complex because the studies reviewed here suggest that executive functions are differentially sensitive to the effect of exercise intensity. Some cognitive processes are impaired at higher intensities (i.e., interference control, response inhibition), some remain fully efficient (i.e., response time), yet others show decrements at lower exercise intensities (working memory). Common across all executive functions is the ability to maintain and manage goals, and use those goals to bias ongoing processing (Friedman and Miyake, 2017). However, the extent to which exercise intensity effects one's ability to use and apply goal representations when engaging inhibition, working memory or cognitive flexibility requires further research. In sum, future work on exercise intensity is needed before claims about specificity or generality of exercise effects on executive functions can be made.

\section{The Impact of Exercise Intensity on Non-executive Functions}

\section{Attention}

Effects of very light to light intensity exercise on attention are mixed (see Table 4). For example, among trained individuals, light-intensity exercise increased errors but did not influence response time on a sustained attention task relative to rest (Radel et al., 2018). Light-intensity exercise did not influence accuracy or response time on a visual oddball task relative to rest (Bullock et al., 2015). However, the remainder of studies measuring very light to light intensity exercise used lighter intensities as their control conditions, rather than using resting conditions as the control, limiting ability to draw conclusions.

Moderate intensity may improve response times, but either impairs or does not affect accuracy on attention tasks when compared to rest. For instance, moderate-intensity exercise speeded response times but increased errors on the SART (Radel et al., 2018). Similarly, moderate intensity speeded response times but reduced accuracy on visual and auditory oddball tasks (Yagi et al., 1999; Bullock et al., 2015). Moderate-intensity exercise increased false alarms on the CPT (Del Giorno et al., 2010) and speeded performance on the PSAT in one study (Quelhas Martins et al., 2013) but not another (Lambourne and Tomporowski, 2010). It speeded alerting, but not orienting or executive control of attention (Huertas et al., 2011). Finally, moderate-intensity exercise decreased response time with no effects on accuracy in two studies using a local global task requiring focusing of visual attention (Pesce et al., 2002), but did not influence speed of visual search (McMorris and Graydon, 1997). Taken together, moderate intensity exercise may improve speed of attentional processing with inconsistent effects on accuracy.

Vigorous to maximal intensities consistently improve attention. Of the six studies to include vigorous- to maximalintensity exercise and rest conditions, four studies found improved response time (McMorris and Graydon, 1997; Pesce et al., 2004; Huertas et al., 2011; Quelhas Martins et al., 2013), with little evidence for impairments (Del Giorno et al., 2010), and one found no differences from rest (Ciria et al., 2019).

\section{Motor Speed}

Across intensities, exercise exerts inconsistent effects on motor speed, specifically reaction time (see Table 1). However certain trends are worth noting and exploring further. Exercise most often speeds reaction time for choice response time, during moderate-intensity exercise and most often slows reaction time for simple reaction time during vigorous-intensity exercise. Relative to rest, very light-intensity exercise has both speeded (Chmura et al., 1998) and not influenced (Davranche and Audiffren, 2004) response time, particularly choice response time. Very light-intensity exercise was also found to slow typing speed (John et al., 2009). Moderate-intensity exercise speeded choice response time across four studies relative to rest (Arcelin et al., 1998; Chmura et al., 1998; Davranche and Audiffren, 2004; Audiffren et al., 2008). Moderate-intensity exercise slowed simple response time in one study, in untrained individuals (Brisswalter et al., 1997). Vigorous-intensity exercise has speeded simple (Collardeau et al., 2001) and choice 
(Paas and Adam, 1991) response time relative to rest. Vigorousintensity exercise has also slowed simple response time in four studies (Brisswalter et al., 1997; Collardeau et al., 2001; Ando et al., 2008, 2010).

\section{Information Processing}

The literature suggests that vigorous-intensity enhances information processing, whereas moderate-intensity may impair it (see Table 6). However, the research is limited to a few studies examining performance at each intensity level, compared to rest. Very light exercise enhanced visual threat detection (Shields et al., 2011). Moderate-intensity exercise improved critical flicker fusion thresholds in one study (Lambourne et al., 2010) but not another (Grego et al., 2004). Vigorous-intensity exercise improved information processing across three studies (Adam et al., 1997; McMorris and Graydon, 1997; Shields et al., 2011) and impaired performance in one study (Paas and Adam, 1991). The one study to evaluate information processing during nearmaximal-intensity exercise found improved performance (McMorris and Graydon, 1997).

\section{Memory}

Across the range of exercise intensities, moderate intensity has most consistently been shown to improve memory, whereas lighter and heavier intensities have demonstrated mixed results (see Table 7). For example, very light and light-intensity exercise did not influence memory (Miles and Hardman, 1998; Hötting et al., 2016; Silvers et al., 2018), whereas moderate-intensity exercise enhanced memory (Grego et al., 2004; Labban and Etnier, 2011; Pyke et al., 2020; Wang et al., 2020). Vigorous intensity-exercise improved memory in five studies (Hötting et al., 2016; van Dongen et al., 2016; Keyan and Bryant, 2017a,b; Loprinzi et al., 2021), and not in five studies (van Dongen et al., 2016; Keyan and Bryant, 2017a; Silvers et al., 2018; Loprinzi et al., 2021). Vigorous-intensity exercise also increased memory interference (Crawford et al., 2021). Similarly, near-maximalintensity exercise both improved and did not influence memory (Frith et al., 2017). The impacts of exercise intensity with regards to duration and time of memory encoding and retrieval are described in section "Findings by Exercise Duration".

\section{Integrative Summary of Intensity Effects on Non-executive Functions}

Within tasks measuring non-executive perceptual-motor functions, such as motor speed and information processing, response time was the most common behavioral dependent variable. Of the studies that assessed speed (response time) as an outcome measure, results generally demonstrated faster information processing across all exercise intensities (very light to vigorous) and improvements in reaction time under moderate to vigorous exercise intensities. Consistent with previous conclusions, reaction time on simple tasks appears to be sensitive to acute exercise, but may not support the inverted-U hypothesis, where moderate intensities would yield greatest improvements. Indeed, previous work has suggested that moderate to vigorous intensity (40-79\% $\mathrm{VO}_{2} \max$ or equivalent) may represent a threshold for improved speed of responding
(McMorris and Hale, 2015). Moreover, there does not appear to be any significant effect of exercise intensity on accuracy of simple cognitive tasks, suggesting that non-executive cognitive processing is not particularly reliable or sensitive as a measure of cognitive performance during acute exercise (McMorris and Hale, 2012; McMorris et al., 2016).

Studies measuring attention during exercise were generally split between those finding improvements and those finding impairments or no effects at each level of exercise intensity. One consistent pattern noted was speeded response times during moderate to maximal exercise intensities, with inconsistent effects on accuracy. Such findings are consistent with studies focusing on motor speed, and suggest that moderate-intensity exercise speeds attentional processes.

Studies measuring memory processes during physical activity suggest that moderate-intensity exercise benefits memory, as does higher-intensity exercise, though less consistently. The memory literature is fairly circumscribed, especially given the variation in exercise intensity and duration and encoding and retrieval timing. Therefore, it is premature to say whether one intensity promotes memory over others, but overall, it seems that exercise is likely to improve memory, with little evidence of deleterious effects. The mechanism by which exercise putatively improves memory may involve increased catecholamine levels and ensuing synaptic plasticity within the hippocampus (Loprinzi, 2018), as well as hippocampal levels of brain-derived neurotrophic factor (BDNF). This interpretation is consistent with previous reviews on exercise and memory, which found that acute exercise exerted moderate to large effects on long-term memory (Roig et al., 2013; but see Loprinzi, 2018 for an example in which high intensity exercise does not impact long-term memory).

\section{Findings by Exercise Duration The Impact of Exercise Duration on Executive Functions \\ Inhibition}

When we investigate the impact of acute exercise on inhibition by duration, response times are enhanced, whereas accuracy is impaired during shorter duration exercise, between 0 and $15 \mathrm{~min}$ (see Table 1). For example, exercise up to $15 \mathrm{~min}$ enhanced response times on both motor response inhibition and interference control tasks across five studies (Davranche et al., 2009, 2015; Ando et al., 2013, 2014; Komiyama et al., 2017). In contrast, five studies demonstrated reduced inhibitory control, with three reporting increased error rates (Pontifex and Hillman, 2007; Ando et al., 2011: Komiyama et al., 2019) and two reporting both increased error rates and slowed response times (McMorris et al., 2009; Smith et al., 2016). Two studies demonstrated no effects (John et al., 2009; Labelle et al., 2013).

When we look at medium duration, exercise lasting 16$30 \mathrm{~min}$ exerts variable effects on response times and accuracy. Both aspects of inhibitory control have been shown to improve and decline in both motor response inhibition and interference control tasks. Specifically, five studies demonstrated improved response times (Davranche and McMorris, 2009; Joyce et al., 2009, 2014; Huertas et al., 2011; Olson et al., 2016; 
Finkenzeller et al., 2019), and one demonstrated combined improvements to both behavioral outcomes (Komiyama et al., 2016). In contrast, three studies demonstrated reduced accuracy, driven by increased error rates (Schmit et al., 2015; Olson et al., 2016; Finkenzeller et al., 2019), with one study demonstrating a more pronounced Simon effect, representing impaired response selection (Davranche and McMorris, 2009). Taken together, it appears acute exercise between 16 and 30 min may improve response time, or impair accuracy, but does not reliably slow response times. However, one study also demonstrated no effects (Huertas et al., 2011). As such, exercise intensity is likely a key factor moderating such changes to performance.

To date, long duration exercise, lasting $31 \mathrm{~min}$ or more, remains relatively unexplored limiting conclusions that can be drawn. More specifically, no studies have examined exercise lasting 31-45 min and two studies have examined performance at durations exceeding $45 \mathrm{~min}$. Enhanced response times with increasing exercise duration was found during $60 \mathrm{~min}$ of vigorous intensity. Yet, reductions in accuracy were demonstrated under both very light and vigorous intensity conditions (Tempest et al., 2017). Conversely, $90 \mathrm{~min}$ of treadmill running at moderate intensity was shown to improve response times, with no change to accuracy, when compared to a light intensity condition (Giles et al., 2018). However, as there is limited work examining inhibition at extended durations, it is difficult to determine whether there is a threshold at which performance may begin to deteriorate.

\section{Working Memory}

Similar to intensity, there are no consistent effects of duration on working memory during exercise (see Table 2). Short-duration exercise, up to $15 \mathrm{~min}$, has been shown to not influence working memory (Komiyama et al., 2017). Exercise lasting 16-30 min primarily resulted in no changes to working memory in four studies (Dutke et al., 2014; Komiyama et al., 2015, 2016, 2019), but improved response times during moderate intensity in one study (Quelhas Martins et al., 2013) and impaired accuracy during vigorous intensity in another (Komiyama et al., 2019). To date, no research has examined aspects of working memory during exercise lasting 31-45 min. Exercise lasting 46-60 min has demonstrated mixed results, with studies finding impaired accuracy (Tempest et al., 2017), enhanced speed (Rattray and Smee, 2013), or no changes in working memory performance (Travlos and Marisi, 1995).

\section{Cognitive Flexibility}

Within studies examining cognitive flexibility, exercise durations longer than 45 min have not yet been examined. At durations of $45 \mathrm{~min}$ or less, the relatively few number of studies and diversity of tasks used to measure varying aspects of cognitive flexibility makes it challenging to compare across studies (see Table 3). Short durations, up to $15 \mathrm{~min}$, differentially influences aspects of cognitive flexibility. For instance, divergent thinking (Oppezzo and Schwartz, 2014) and ability to flexibly switch focus of attention from local to global (Pesce et al., 2003, 2007) may be improved during shorter bouts of exercise, whereas convergent thinking (Oppezzo and Schwartz, 2014) and task-switching may be impaired (Labelle et al., 2013). To note, these studies did not specifically report exercise duration and times were estimated from information reported in the methods. Exercise lasting 16-30 min resulted in impairments in accuracy on the WCST under vigorous intensity (Del Giorno et al., 2010; Wang et al., 2013), no differences under moderate intensity (Wang et al., 2013), and mixed results under light intensity (Del Giorno et al., 2010; Wang et al., 2013). Longer duration exercise, lasting 31-45 min impaired accuracy on the WCST at vigorous intensity (Dietrich and Sparling, 2004). More work is needed to specifically determine how factors such as intensity or duration may influence aspects of flexibility, such as perseveration and set-shifting, task-switching and switching focus on attention, as well as convergent and divergent thinking.

\section{Integrative Summary of Duration Effects on Executive Functions}

Similar to exercise intensity, exercise duration moderates executive function during exercise. Here we find negative effects for inhibition tasks performed between 0 and $15 \mathrm{~min}$ of exercise. These results were specific to accuracy, whereas response times improved up to $30 \mathrm{~min}$, and declined past $60 \mathrm{~min}$. These findings generally align with previous meta-analytic reviews demonstrating detrimental or negligible effects between min 11$20 \mathrm{~min}$ and beneficial effects after $20 \mathrm{~min}$ of exercise (Lambourne and Tomporowski, 2010; Chang et al., 2012). Given the few studies exploring cognitive flexibility and working memory across varying exercise durations, conclusions are still limited.

\section{The Impact of Exercise Duration on Non-executive Functions Attention}

Exercise duration does not appear to consistently influence attention during exercise (see Table 4). Short-duration exercise, up to $15 \mathrm{~min}$, enhanced aspects of attention in six studies (McMorris and Graydon, 1997; Yagi et al., 1999; Pesce et al., 2002, 2004; González-Fernández et al., 2017), impaired in one study (Wohlwend et al., 2017), and did not influence attention in two studies (McMorris and Graydon, 1997; Yagi et al., 1999), Exercise lasting 16-30 min improved attention in three studies (Huertas et al., 2011; Sanabria et al., 2011; Hüttermann and Memmert, 2014), impaired attention in three studies (Del Giorno et al., 2010; Hüttermann and Memmert, 2014; Cortney Bradford et al., 2019), and did not influence attention in two studies (Hüttermann and Memmert, 2014; Ciria et al., 2019). Exercise lasting 31-45 min enhanced speed of attention when moderate in intensity (Bullock et al., 2015; Radel et al., 2017, 2018; Sanchis et al., 2020), but increased errors when light and moderate in intensity (Radel et al., 2018). In contrast, one study demonstrated no influence on attention (Lambourne et al., 2010).

\section{Motor Speed}

During moderate-intensity exercise, and exercise durations between 15 and $90 \mathrm{~min}$, we see that exercise most often speeds reaction time for choice response time. Exercise most often slows reaction time for simple reaction time, for vigorous-intensity 
exercise, and very short (less than $15 \mathrm{~min}$ ) and long (more than $90 \mathrm{~min}$ ) durations (see Table 5). The majority of studies used durations less than $15 \mathrm{~min}$. Of these, one found speeded choice response time (Arcelin et al., 1998), three found slowed simple response times (Brisswalter et al., 1997; Ando et al., 2008, 2010), and one found no difference (Arcelin and Brisswalter, 1999). Of the three studies of 15-30 min exercise duration, two found speeded choice response times (Paas and Adam, 1991; Davranche and Audiffren, 2004), and one found no difference (Davranche and Audiffren, 2004).

\section{Information Processing}

Performance on perceptual tasks have only been assessed across a few exercise durations, limiting conclusions (see Table 6). Exercise between 20 and 40 min exerts variable effects on information processing, enhancing performance in three studies (Adam et al., 1997; Lambourne et al., 2010; Shields et al., 2011), but impairing in one (Paas and Adam, 1991). Exercise of longer durations, particularly in the second and third hour of exercise of $180 \mathrm{~min}$ durations, impaired information processing (Grego et al., 2004; see Table 2). However, to date, durations less than $20 \mathrm{~min}$ and between 40 and $180 \mathrm{~min}$ remain unexplored.

\section{Memory}

Memory appears to be improved or unaffected across the spectrum of exercise durations (see Table 7). In addition to intensity and duration, the timing of encoding and retrieval is essential to consider. Encoding occurred from 2 days before exercise to during exercise, and retrieval occurred during exercise to 1 week after exercise.

Ten studies evaluated the influence of exercise on encoding before exercise and retrieval during or after exercise. For encoding 2 days before exercise, 20-25 min of vigorous-intensity exercise enhanced memory for central details 2 days following exercise when memory was reactivated during exercise (Keyan and Bryant, 2017a). Exercise did not influence memory when not reactivated, nor for peripheral details or intrusive memories. Thirty five minutes of vigorous-intensity exercise improved cued recall 2 days after exercise for information encoded 4 $\mathrm{h}$ before exercise, but not immediately before exercise (van Dongen et al., 2016). For encoding $10 \mathrm{~min}$ before exercise, $30 \mathrm{~min}$ of vigorous-intensity exercise enhanced memory $24 \mathrm{~h}$ after exercise, but not 20 min after exercise (Hötting et al., 2016). The same study found no effects of very light to light-intensity exercise on memory.

For encoding immediately before exercise, $10 \mathrm{~min}$ of very light- to vigorous-intensity exercise did not influence cued recall 2 days after exercise, but increased intrusive memories (Keyan and Bryant, 2017b). Thirty minutes of moderate- to vigorousintensity enhanced verbal memory 35 and $60 \mathrm{~min}$ as well as $24 \mathrm{~h}$ after encoding (Labban and Etnier, 2011; Wang et al., 2020) and old/new recognition 80-90 min after encoding (Pyke et al., 2020). Fifteen minutes of near-maximal-intensity, short-duration exercise enhanced 20-min and 24-h delayed verbal memory as well as prospective memory when performed before encoding, but not during or after encoding (Frith et al., 2017). Fifteen minutes of vigorous-intensity exercise also increased memory interference $5 \mathrm{~min}$ after exercise, relative to rest (Crawford et al., 2021). Twenty minutes vigorous-intensity exercise did not influence incidental or intentional encoding immediately or $30 \mathrm{~min}$ after exercise (Loprinzi et al., 2021), but enhanced verbal recall through encoding and retrieval in Experiment 1 and consolidation in Experiment 2 (Loprinzi et al., 2021).

In addition to Frith et al. (2017) described above, three studies evaluated the influence of encoding during exercise and retrieval during or after exercise. Moderate-intensity exercise improved map recognition between the first and second of $3 \mathrm{~h}$ exercise (Grego et al., 2004). Verbal memory was better when encoded and retrieved during $11 \mathrm{~min}$ exercise and when encoding and retrieved during rest than when encoding during exercise and retrieved during rest and vice-versa, providing evidence for state-dependent learning (Miles and Hardman, 1998). Finally, 20 min very light-, light-, and vigorous-intensity exercise did not influence immediate or 1-week delayed memory (Silvers et al., 2018).

\section{Integrative Summary of Duration Effects on Non-executive Functions}

The majority of studies focusing motor speed show speeded responses on SRT, particularly after 15 min of exercise. Likewise, exercise improves information processes after 20 min of exercise, but performance declined after 2 and $3 \mathrm{~h}$ of exercise. On the other hand, exercise has been generally shown to improve attentional processes up to $15 \mathrm{~min}$ of exercise, after which the effects become more variable. Overall, it appears attentional processes improve very early in the exercise bout, whereas perceptualmotor processes improve after some time. However, more work spanning short to long durations is need to determine specific time points at which perceptual-motor and attentional processes are impacted.

For memory, exercise duration as well as the timing of encoding and retrieval are essential to consider in terms of sequence of events. A recent meta-analysis encompassing the present articles, as well as those in which exercise occurred prior to encoding, suggests that although exercise during memory encoding did not influence retrieval, short-duration exercise tended to impair memory relative to the control (Loprinzi, 2019). Exercise consolidation enhanced episodic memory, particularly for long-duration exercise during early consolidation, and shortduration exercise during late consolidation. Around the time of that meta-analysis, a handful of studies have begun to better disentangle the effects of encoding and retrieval timing by administering encoding before, during, and after exercise, and retrieval a relatively short and long while after exercise (Frith et al., 2017; Pyke et al., 2020; Loprinzi et al., 2021). These studies have broadly suggested that the mechanism by which exercise enhances memory may work through encoding, consolidation, and retrieval. Future research should take a similar approach, but also systematically vary duration and intensity. At present, the literature suggests that exercising between learning and retrieving information improves memory at best, and does not influence memory at worse. Given exercise's benefits to stress and mood (Basso and Suzuki, 2017), it is likely to benefit learning contexts. 


\section{WHAT IS NEXT?}

As we have seen in the preceding sections, the impact of exercise on executive and non-executive processes varies dramatically depending on the specific cognitive domain, as well as by intensity and duration. In the following section, we discuss several moderating factors that may contribute to these mixed findings, highlight existing gaps in our knowledge and propose future directions for work in this field.

\section{Factors That Impact Cognition During Exercise \\ Participant Characteristics \\ Fitness Level}

Certain participant characteristics may affect cognition during exercise. One important characteristic to consider is participant's fitness level. To date, studies have compared young adults of varying fitness levels (see Supplementary Table $\mathbf{1}$ for participant characteristics for all studies). For example, studies examining the impacts of fitness on executive functions found that exercise did not influence inhibition among higher- or lowerfit individuals, but increased error rate more so in lower- than higher-fit individuals (Labelle et al., 2013). Interestingly, majority of studies examining motor and perceptual processes during exercise have used physically fit individuals. For example, within studies assessing motor speed, nearly all extant studies included physically fit individuals, whose $\mathrm{VO}_{2}$ max averages fell above the 50th percentile, often above the 75th percentile (Kaminsky et al., 2015). The one study to compare simple response time during exercise between trained and untrained individuals found that exercise at all intensities ranging from light to vigorous slowed simple response time in untrained individuals, but this effect dissipated at moderate and vigorous-intensity exercise in trained individuals (Brisswalter et al., 1997). Indeed, populations such as endurance athletes may differ from the general population. However, to date still few studies have compared cognitive function during exercise between lower and higher fit individuals, or between sedentary individuals and/or athletes of varying fitness levels. Thus, future work should consider how this participant characteristic may guide or impact research questions.

\section{Psychological Factors}

Future work should also consider the psychological factors that are at play in realistic exercise scenarios (e.g., anticipatory anxiety before athletic performance, cognitive stress during military operations) that may influence motivation in lab-based research. For example, acute anxiety experienced during exercise has been shown to mitigate declines in inhibitory control under long duration, high intensity exercise (Cantelon et al., 2019). Additionally, research has demonstrated that mental resource allocation, perception of effort and prefrontal cortex activation are differentially affected when exercise end-point is known vs. unknown (Radel et al., 2017; Wingfield et al., 2019), yet it remains unknown how such anticipation may influence cognitive function during exercise. Given that a motivating factor for much of the research in this field is to characterize performance decrements that could lead to costly performance outcomes (e.g., game-losing play, or life or death decisions), basic work should seek to emulate the emotional and motivational factors that may influence performance in applied settings.

\section{Methodological Factors}

\section{Dependent Outcome Measures: Speed vs. Accuracy}

Another factor that contributes to the heterogeneous patterns of results observed within the acute exercise-cognition literature is the dependent outcome variable measured. Speed (response time) and accuracy of cognitive performance tend to show differential patterns of results. For example, improvements to inhibition are largely driven by faster responses, whereas decrements are driven by changes in response accuracy. Previous meta-analyses have demonstrated similar effects, such that when accuracy was the dependent variable, results were significantly different to those when response time was the dependent variable (McMorris et al., 2011; McMorris and Hale, 2012). Inconsistent findings with regards to accuracy within the exercise-cognition literature may be driven by use of cognitive tasks primarily designed to measure speed of processing (i.e., flanker, simple, go/no-go, simple, and choice reaction time) (McMorris, 2016), or the result of an inability to detect exercise-induced changes due to ceiling effects often demonstrated in the healthy young populations sampled (McMorris and Hale, 2012). However, given that response time is more consistently influenced than accuracy across various cognitive domains, interpretations are limited. It is possible that different mechanisms may be contributing to the changes to response time or accuracy during exercise (McMorris and Hale, 2015). Thus, if changes to response times or accuracy are elicited at distinct or differing physiological thresholds, ability to adequately assess changes to both speed and accuracy in a single study may be limited. Future work should continue to explore these differential effects on response time and accuracy, as ability to detect even slight deteriorations of such processes could allow us to better predict potential negative outcomes (life or death, losing play in a game).

\section{Exercise Modality}

Exercise modality has also been shown to differentially impact cognition during exercise. For example, within the executive domain, studies demonstrating impairments in working memory used treadmill, while those demonstrating improvements or no differences employed cycle ergometry. Previous meta-analytic reviews have revealed that exercise modality is an important factor in determining cognitive function during exercise, where running was shown to be related to declines in performance and cycling with improvements (Lambourne and Tomporowski, 2010). Inconsistent results based on exercise modality may be attributable to differences in the physical effort required during running vs. cycling. For instance, walking and running require balance and control of body posture. Thus, negative effects of simultaneous exercise on, say, working memory, may be due to the attentional conflict between coordination of bodily movement and executive control. Performing cognitive tasks during exercise inherently creates a dual-task environment, but these dual-task effects may be more pronounced depending on the exercise modality (see Supplementary Table 1 for exercise modality used in all studies in this review). However, given that 
the vast majority of studies utilize cycling compared to running, walking, or other forms of aerobic exercise, more research is needed to further establish whether specific relationships exist between exercise modality, intensity and cognitive domains.

\section{Additional Gaps and Future Directions Unified Methods for Prescribing Intensity}

Exercise intensity is often considered the most important component of exercise prescription as it represents the magnitude of metabolic stress on the body. Poor quantification or inadequate control of intensity likely contributes to the heterogeneity of results in exercise-cognition relationships. Furthermore, prescription and categorization of exercise intensity often varies from study to study. For example, 60\% HRmax may be classified by authors as moderate, whereas using the ACSM guidelines followed here, this intensity is considered to be within the light range. Hence, the field needs unified methods so results can be more easily compared across studies. It has been argued that intensity should be determined in relation to the aerobic threshold, which might allow us to better understand the physiological and biochemical factors that contribute to changes during exercise. This may also confer better understanding of exercise intensity effects on cognition for exercise prescription. Relatedly, cognitive testing at rest, either before exercise or during a separate resting day, is essential to interpret results across studies, and as such, future studies should aim to test cognition at rest compared to during exercise.

\section{Intensity Gaps}

As evidenced in section ("What Has Been Done?"), research spanning the matrix of exercise intensities and durations is incomplete (see Figure 1). Notably, across all cognitive domains, very few studies have explored performance under maximal effort conditions. This extreme exercise intensity poses methodological challenges, however, understanding how cognition is impacted under such conditions may have applied relevance for certain populations (i.e., military personnel or athletes). Such examination may provide insights into when and/or how fatigue leads to declines in cognitive performance.

In line with this notion, future work should continue to examine how cognitive performance may change at, or near, physiological transition points. For example, it appears that inhibition accuracy may begin to decline above the ventilatory threshold (VT). Indeed, reduced accuracy has been demonstrated in studies prescribing target intensities at vigorous, or near the upper boundary of the moderate intensity range [i.e., $60 \%$ $\mathrm{VO}_{2}$ peak; 60\% MAP ( 70-82\% HRmax)] (Garber et al., 2011; Olson et al., 2016; Finkenzeller et al., 2019) and in previous work using an incremental exercise protocol (Da Silva et al., 2017). In these studies, reduced accuracy was driven by inability to avoid strong prepotent responses, or distraction from taskirrelevant information. Thus, one thought is that increasing exercise intensity may reduce the ability to effectively maintain and use goal representations to bias competition from conflicting information. Higher levels of physical stress leads to shifts in attentional deployment, with increased internal (associative) focus of attention at the expense of attentional resources available for external (dissociative) focus (Tenenbaum, 2007). Research has demonstrated that at intensities above VT individuals often report increases in associative thoughts relating to physical sensations, or interoceptive cues, that accompany the metabolic changes due to exercise (i.e., breathing rhythm, muscle fatigue, heart rate, and temperature) (Ekkekakis et al., 2011; Da Silva et al., 2017). These attentional shifts mirror the theorized redistribution of resources from PFC to motor areas, as well as observed activation of regions associated with autonomic regulation (i.e., insular cortex) demonstrated at increasing exercise intensities (Dietrich and Audiffren, 2011; Audiffren, 2016; Fontes et al., 2019). Thus, future work should examine whether specific physiological thresholds must be exceeded before declines in cognitive function are revealed, and specifically where accuracy of executive functions may begin to deteriorate.

\section{Duration Gaps}

Future work should also look to fill the gaps in our understanding of exercise duration, particularly for endurance exercise. Changes in cognition over relatively short durations are pertinent to the general population aiming to meet the Physical Activity Guidelines (American College of Sports Medicine, 2018), or athletes who perform in discrete playing periods. However, changes in cognition during longer duration exercise are essential to understand for endurance athletes as well as military personnel and emergency responders, who must remain cognitively intact in prolonged, physically demanding situations. While many studies look at cognition as a function of exercise intensity, very few do so for exercise duration. To date, this area of the field remains largely unexplored, as evidenced in Figure 1, where the majority of studies focus on durations of 45 min or less.

Furthermore, the lack of systematic and consistent findings with regards to longer exercise durations may be due to timing of data sampling. Time-averaged cognitive performance does not fully capture potential temporal dynamics of cognitive functioning throughout the exercise bout. For example, during a prolonged bout performance may increase or decrease at varying time points. As such, new theoretical perspectives have been proposed to explain how top-down (cognitive and physical efforts) and bottom-up processes (bodily sensations)

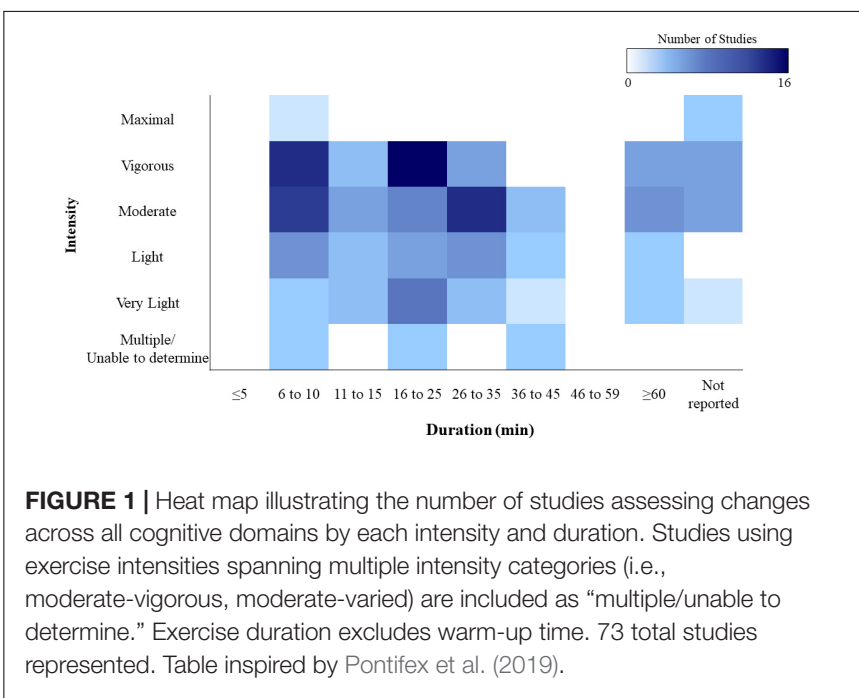


may act in parallel of arousing mechanisms to dynamically influence cognitive performance across time (see Schmit and Brisswalter, 2018 for review of this fatigue-based neurocognitive perspective). However, given the scarce number of studies investigating performance at durations extending beyond $45 \mathrm{~min}$, theoretical predictions and current understanding of timedependent changes remain understudied.

Finally specific reporting of duration parameters will improve our ability to draw conclusions about specific effects due to exercise duration. For instance, within the domain of attention, majority of experiments performed did not report total exercise duration. Lack of reporting duration parameters, including total time spent exercising and minutes spent in warm-up and at prescribed intensities, as well as specific timing of when cognitive tasks were administered is problematic and makes it difficult or impossible to interpret results on how duration impacts cognition. Similar to developing unified methods for determining exercise intensity, common methodological factors should be reported across future work.

\section{Statistical Quantification of Exercise Effects}

Finally, the narrative nature of this review allowed for a more nuanced exploration of how cognition is impacted during exercise that has potentially been overlooked in prior research. For instance, Chang et al. (2012) concluded that exercise intensity does not influence cognitive performance, but here we see that intensity-dependent effects may depend on the cognitive task type, exercise duration and/or fitness level. These complex effects are difficult to capture using meta-analytic techniques. However, lack of systematic and quantitative comparison also limits the conclusions that can be drawn. Here, we aimed to highlight potential areas where future work may be useful in order to enhance conclusions that can be drawn from meta-analysis. Thus, as empirical work examining cognitive changes during exercise grows, new meta-analytic reviews will be essential in identifying reliable exercise-induced effects.

\section{Limitations}

The present review only included studies prescribing aerobic exercise. Yet, the majority of empirical studies measuring cognition during exercise often employ bouts of aerobic activity. This may be due to the fact that previous work in this field has largely been driven by understanding and enhancing performance of athletes, as well as law enforcement and military personnel, who often operate under such aerobically demanding conditions. However, given that alternate forms of exercise (i.e., HIIT, resistance, coordinative, etc.) are gaining popularity, both within the general public and research community, it is important for future work to characterize exercise-cognition interactions beyond aerobic exercise. Specifically, understanding cognitive changes elicited at physiological thresholds may be well-suited for incremental or HIIT exercise protocols, where strictly controlled exercise intensities allow for more precise measurement of intensity-induced changes.

In depth examination of underlying neural mechanisms facilitating cognitive changes during acute bouts of exercise was not within the scope of this review. Additionally, not all of the studies reviewed here conducted objective measurement of the mechanisms involved in the exercise effects reported. However, characterizing how underlying neural changes influence cognition, such as changes in cerebral blood flow or electrical potential, is important and will be necessary in allowing the field to further develop and refine current theories. As future research continues to explore exercise-cognition interactions, it will be important to tie neurological and physiological mechanisms to changes in cognitive function.

\section{CONCLUSION}

The current review summarized the critical characteristics of literature examining cognitive changes during acute bouts of aerobic exercise. First, we characterized what aspects of cognition have been explored during exercise and common cognitive tasks used. Across cognitive domains, we find more evidence for exercise impacting speed over accuracy of responding. Future work should consider how choice of cognitive tasks and populations sampled may impact ability to detect changes in behavioral outcomes of interest. In line with this notion, we suggest that adopting standardized methods of prescribing and reporting exercise parameters would be advantageous for the field.

Next, to date, extant literature has largely focused on examining one sub-component of executive function during exercise, namely inhibition. Working memory and cognitive flexibility are two other important components of executive function. Conclusions about how inhibition is impacted during exercise might not generalize to working memory and cognitive flexibility. Regardless of executive or non-executive domain, under shorter durations and light to moderate intensities cognitive performance may not be drastically impacted. Higher intensity and longer duration exercise may impair certain aspects of cognition, but literature in this area remains sparse. Finally, information presented here may provide translational application for sports performance or individuals working under states of physical exertion, such as endurance athletes or first responders and military personnel.

Overall, the effects on cognition during exercise are likely mixed due to methodological differences referenced above, but also because exercise exerts its effects through multiple mechanisms. Research should continue to characterize cognitive changes during exercise, as well as the mechanisms that drive such changes, in order to refine and develop the theories of exercise-induced changes to cognition. This will help us to develop tools to predict cognitive changes during physical exertion.

\section{AUTHOR CONTRIBUTIONS}

JC and GG were responsible for conceptualizing and designing the review and interpreting relevant literature. JC was responsible for write-up of the manuscript. Both authors contributed to manuscript revision, as well as read and approved the final manuscript. 


\section{FUNDING}

This work was funded by the U.S. Army DEVCOM Soldier Center.

\section{ACKNOWLEDGMENTS}

The views expressed in this article are solely those of the authors and do not reflect the official policies or positions of the

\section{REFERENCES}

Adam, J. J., Teeken, J. C., Ypelaar, P. J. C., Verstappen, F. T. J., and Paas, F. G. W. (1997). Exercise-induced arousal and information processing. Int. J. Sport Psychol. 28, 217-226.

Alvarez, J. A., and Emory, E. (2006). Executive function and the frontal lobes: a meta-analytic review. Neuropsychol. Rev. 16, 17-42. doi: 10.1007/s11065-0069002-x

American College of Sports Medicine (2018). ACSM's Exercise Testing and Prescription. Philadelphia, PA: Lippincott williams \& wilkins.

Ando, S., Hatamoto, Y., Sudo, M., Kiyonaga, A., Tanaka, H., and Higaki, Y. (2013). The effects of exercise under hypoxia on cognitive function. PLoS One 8:e63630. doi: 10.1371/journal.pone.0063630

Ando, S., Kokubu, M., Kimura, T., Moritani, T., and Araki, M. (2008). Effects of acute exercise on visual reaction time. Int. J. Sports Med. 29, 994-998.

Ando, S., Kokubu, M., Yamada, Y., and Kimura, M. (2011). Does cerebral oxygenation affect cognitive function during exercise? Eur. J. Appl. Physiol. 111, 1973-1982. doi: 10.1007/s00421-011-1827-1

Ando, S., Komiyama, T., Sudo, M., and Higaki, Y. (2014). The effects of acute exercise and hypoxia on cognitive function. Int. J. Psychophysiol. 94:135. doi: 10.1016/j.ijpsycho.2014.08.631

Ando, S., Yamada, Y., and Kokubu, M. (2010). Reaction time to peripheral visual stimuli during exercise under hypoxia. J. Appl. Physiol. 108, 1210-1216. doi: 10.1152/japplphysiol.01115.2009

Arcelin, R., and Brisswalter, J. (1999). Performance stability in simultaneous tasks of pedalling and reaction time. Percept. Mot. Skills 88, 1193-1199. doi: 10.2466/ PMS.88.3.1193-1199

Arcelin, R., Delignieres, D., and Brisswalter, J. (1998). Selective effects of physical exercise on choice reaction processes. Percept. Mot. Skills 87, 175-185. doi: 10.2466/pms.1998.87.1.175

Arts, F. J., and Kuipers, H. (1994). The relation between power output, oxygen uptake and heart rate in male athletes. Int. J. Sports Med. 15, 228-231. doi: $10.1055 / \mathrm{s}-2007-1021051$

Audiffren, M. (2016). The reticular-activating hypofrontality (RAH) model of acute exercise: current data and future perspectives. Exerc. Cogn. Interact. 2, 436-447. doi: 10.1016/B978-0-12-800778-5.00007-4

Audiffren, M., Tomporowski, P. D., and Zagrodnik, J. (2008). Acute aerobic exercise and information processing: energizing motor processes during a choice reaction time task. Acta Psychol. 129, 410-419. doi: 10.1016/j.actpsy. 2008.09.006

Basso, J. C., and Suzuki, W. A. (2017). The effects of acute exercise on mood, cognition, neurophysiology, and neurochemical pathways: a review. Brain Plast. 2, 127-152. doi: 10.3233/BPL- 160040

Borg, G. A. (1982). Psychophysical bases of perceived exertion. Med. Sci. Sports Exerc. 14, 377-381. doi: 10.1249/00005768-198205000-00012

Brisswalter, J., Arcelin, R., Audiffren, M., and Delignieres, D. (1997). Influence of physical exercise on simple reaction time: effect of physical fitness. Percept. Mot. Skills 85(3 Pt 1), 1019-1027. doi: 10.2466/pms.1997.85.3. 1019

Brisswalter, J., Callardeau, M., and René, A. (2002). Effects of acute physical exercise on cognitive performance. Sports Med. 32, 555-566. doi: 10.1097/00005768199701000-00009
Department of Army, the Department of Defense, or any other department or agency of the U.S. Government.

\section{SUPPLEMENTARY MATERIAL}

The Supplementary Material for this article can be found online at: https://www.frontiersin.org/articles/10.3389/fpsyg. 2021.653158/full\#supplementary-material

Supplementary Table 1 | Complete summary of studies included in this review.

Bullock, T., Cecotti, H., and Giesbrecht, B. (2015). Multiple stages of information processing are modulated during acute bouts of exercise. Neuroscience 307, 138-150. doi: 10.1016/j.neuroscience.2015.08.046

Cantelon, J. A., Giles, G. E., Eddy, M. D., Haga, Z., Mahoney, C. R., Taylor, H. A., et al. (2019). Exerting cognitive control under threat: interactive effects of physical and emotional stress. Emotion 19, 1236-1243. doi: 10.1037/ emo0000509

Chang, Y. K. K., Labban, J. D. D., Gapin, J. I. I., and Etnier, J. L. L. (2012). The effects of acute exercise on cognitive performance: a meta-analysis. Brain Res. 1453, 87-101. doi: 10.1016/J.BRAINRES.2012.02.068

Chmura, J., Krysztofiak, H., Ziemba, A. W., Nazar, K., and Kaciuba-Uscilko, H. (1998). Psychomotor performance during prolonged exercise above and below the blood lactate threshold. Eur. J. Appl. Physiol. Occup. Physiol. 77, 77-80. doi: 10.1007/s004210050303

Chun, M. M. (2000). Contextual cueing of visual attention. Trends Cogn. Sci. 4, 170-178. doi: 10.1016/S1364-6613(00)01476-5

Ciria, L. F., Luque-Casado, A., Sanabria, D., Holgado, D., Ivanov, P. C., and Perakakis, P. (2019). Oscillatory brain activity during acute exercise: tonic and transient neural response to an oddball task. Psychophysiology 56:e13326. doi: 10.1111 psyp. 13326

Collardeau, M., Brisswalter, J., Vercruyssen, F., Audiffren, M., and Goubault, C. (2001). Single and choice reaction time during prolonged exercise in trained subjects: influence of carbohydrate availability. Eur. J. Appl. Physiol. 86, 150156. doi: $10.1007 / \mathrm{s} 004210100513$

Cortney Bradford, J., Lukos, J. R., Passaro, A., Ries, A., and Ferris, D. P. (2019). Effect of locomotor demands on cognitive processing. Sci. Rep. 9, 1-12. doi: 10.1038/s41598-019-45396-5

Crawford, L. K., Caplan, J. B., and Loprinzi, P. D. (2021). The impact of acute exercise timing on memory interference. Percept. Mot. Skills 128, 1215-1234. doi: 10.1177/0031512521993706

Crowder, R. (2015). Principles of Learning and Memory: Classic Edition. New York, NY: Psychology Press.

Da Silva, W. Q. A., Fontes, E. B., Forti, R. M., Lima, Z. L., Machado, D. G. D. S., Deslandes, A. C., et al. (2017). Affect during incremental exercise: the role of inhibitory cognition, autonomic cardiac function, and cerebral oxygenation. PLoS One 12:e0186926. doi: 10.1371/journal.pone.0186926

Davranche, K., and Audiffren, M. (2004). Facilitating effects of exercise on information processing. J. Sports Sci. 22, 419-428. doi: 10.1080/ 02640410410001675289

Davranche, K., Brisswalter, J., and Radel, R. (2015). Where are the limits of the effects of exercise intensity on cognitive control? J. Sport Health Sci. 4, 56-63. doi: 10.1016/j.jshs.2014.08.004

Davranche, K., Burle, B., Audiffren, M., and Hasbroucq, T. (2005). Information processing during physical exercise: a chronometric and electromyographic study. Exp. Brain Res. 165, 532-540. doi: 10.1007/s00221-005-2331-9

Davranche, K., Burle, B., Audiffren, M., and Hasbroucq, T. (2006). Physical exercise facilitates motor processes in simple reaction time performance: an electromyographic analysis. Neurosci. Lett. 396, 54-56. doi: 10.1016/J. NEULET.2005.11.008

Davranche, K., Hall, B., and McMorris, T. (2009). Effect of acute exercise on cognitive control required during an Eriksen flanker task. J. Sport Exerc. Psychol. 31, 628-639. 
Davranche, K., and McMorris, T. (2009). Specific effects of acute moderate exercise on cognitive control. Brain Cogn. 69, 565-570. doi: 10.1016/j.bandc.2008. 12.001

Del Giorno, J. M., Hall, E. E., O’Leary, K. C., Bixby, W. R., and Miller, P. C. (2010). Cognitive function during acute exercise: a test of the transient hypofrontality theory. J. Sport Exerc. Psychol. 32, 312-323. doi: 10.1123/jsep.32.3.312

Diamond, A. (2012). Executive functions. Annu. Rev. Psychol. 64, 135-168. doi: 10.1146/annurev-psych-113011-143750

Dietrich, A. (2003). Functional neuroanatomy of altered states of consciousness: the transient hypofrontality hypothesis. Conscious. Cogn. 12, 231-256. doi: 10.1016/s1053-8100(02)00046-6

Dietrich, A. (2006). Transient hypofrontality as a mechanism for the psychological effects of exercise. Psychiatry Res. 145, 79-83. doi: 10.1016/j.psychres.2005.07. 033

Dietrich, A., and Audiffren, M. (2011). The reticular-activating hypofrontality (RAH) model of acute exercise. Neurosci. Biobehav. Rev. 35, 1305-1325. doi: 10.1016/j.neubiorev.2011.02.001

Dietrich, A., and Sparling, P. B. (2004). Endurance exercise selectively impairs prefrontal-dependent cognition. Brain Cogn. 55, 516-524. doi: 10.1016/j.bandc. 2004.03.002

Dinges, D., and Powell, J. (1985). Microcomputer analyses of performance on a portable, simple visual RT task during sustained operations. Behav. Res. Methods Instrum. Comput. 17, 652-655. doi: 10.1080/19475683.2010.513153

Donders, F. C. (1969). On the speed of mental processes. Acta Psychol. 30, 412-431. doi: 10.1016/0001-6918(69)90065-1

Dutke, S., Jaitner, T., Berse, T., and Barenberg, J. (2014). Acute physical exercise affected processing efficiency in an auditory attention task more than processing effectiveness. J. Sport Exerc. Psychol. 36, 69-79. doi: 10.1123/jsep.20130044

Easterbrook, J. A. (1959). The effect of emotion on cue utilization and the organization of behavior. Psychol. Rev. 66, 183-201. doi: 10.1037/h0047707

Ekkekakis, P., Parfitt, G., and Petruzzello, S. J. (2011). The pleasure and displeasure people feel when they exercise at different intensities: decennial update and progress towards a tripartite rationale for exercise intensity prescription. Sports Med. 41, 641-671. doi: 10.2165/11590680-000000000-00000

Erickson, K. I., Hillman, C., Stillman, C. M., Ballard, R. M., Bloodgood, B., Conroy, D. E., et al. (2019). Physical activity, cognition, and brain outcomes: a review of the 2018 physical activity guidelines. Med. Sci. Sports Exerc. 51, 1242-1251. doi: 10.1249/MSS.0000000000001936

Eriksen, B. A., and Eriksen, C. W. (1974). Effects of noise letters upon the identification of a target letter in a non-search task. Attent. Percept. Psychophys. 16, 143-149. doi: 10.3758/BF03203267

Fan, J., McCandliss, B. D., Sommer, T., Raz, A., and Posner, M. I. (2002). Testing the efficiency and independence of attentional networks. J. Cogn. Neurosci. 14, 340-347. doi: 10.1162/089892902317361886

Finkenzeller, T., Würth, S., Doppelmayr, M., and Amesberger, G. (2019). Temporal dynamics of varying physical loads on speed and accuracy of cognitive control. J. Sport Exerc. Psychol. 41, 206-214. doi: 10.1123/jsep.20180239

Fontes, E. B., Bortolotti, H., Grandjean da Costa, K., Machado de Campos, B., Castanho, G. K., Hohl, R., et al. (2019). Modulation of cortical and subcortical brain areas at low and high exercise intensities. Br. J. Sports Med. 54, 110-115. doi: 10.1136/bjsports-2018-100295

Friedman, N. P., and Miyake, A. (2004). The relations among inhibition and interference control functions: a latent-variable analysis. J. Exp. Psychol. 133, 101-135. doi: 10.1037/0096-3445.133.1.101

Friedman, N. P., and Miyake, A. (2017). Unity and diversity of executive functions: individual differences as a window on cognitive structure. Cortex 86, 186-204. doi: 10.1016/j.cortex.2016.04.023

Frith, E., Sng, E., and Loprinzi, P. D. (2017). Randomized controlled trial evaluating the temporal effects of high-intensity exercise on learning, short-term and long-term memory, and prospective memory. Eur. J. Neurosci. 46, 2557-2564. doi: $10.1111 /$ ejn. 13719

Garber, C. E., Blissmer, B., Deschenes, M. R., Franklin, B. A., Lamonte, M. J., and Lee, I. M. (2011). Quantity and quality of exercise for developing and maintaining cardiorespiratory, musculoskeletal, and neuromotor fitness in apparently healthy adults: guidance for prescribing exercise. Med. Sci. Sports Exerc. 43, 1334-1359. doi: 10.1249/MSS.0b013e318213fefb
Giles, G. E., Eddy, M. D., Brunyé, T. T., Urry, H. L., Graber, H. L., Barbour, R. L., et al. (2018). Endurance exercise enhances emotional valence and emotion regulation. Front. Hum. Neurosci. 12:398. doi: 10.3389/fnhum.2018.00398

González-Fernández, F., Etnier, J. L., Ab Ala, M. Z., and Sanabria, D. (2017). Vigilance performance during acute exercise. Int. J. Sport Psychol. 48, 435-447. doi: 10.7352/IJSP2017.48.435

Grego, F., Vallier, J. M., Collardeau, M., Bermon, S., Ferrari, P., Candito, M., et al. (2004). Effects of long duration exercise on cognitive function, blood glucose, and counterregulatory hormones in male cyclists. Neurosci. Lett. 364, 76-80. doi: 10.1016/j.neulet.2004.03.085

Gronwall, D. M. (1977). Paced auditory serial-addition task: a measure of recovery from concussion. Percept. Mot. Skills 44, 367-373. doi: 10.2466/pms.1977.44.2. 367

Harvey, P. D. (2019). Domains of cognition and their assessment. Dialogues Clin. Neurosci. 21, 227-237. doi: 10.31887/dens.2019.21.3/pharvey

Herrmann, C. S., and Knight, R. T. (2001). Mechanisms of human attention: event-related potentials and oscillations. Neurosci. Biobehav. Rev. 25, 465-476. doi: 10.1016/s0149-7634(01)00027-6

Hötting, K., Schickert, N., Kaiser, J., Röder, B., and Schmidt-Kassow, M. (2016). The effects of acute physical exercise on memory, peripheral BDNF, and cortisol in young adults. Neural Plast. 2016, 1-12. doi: 10.1155/2016/6860573

Huertas, F., Zahonero, J., Sanabria, D., and Lupiáñez, J. (2011). Functioning of the attentional networks at rest vs. during acute bouts of aerobic exercise. J. Sport Exerc. Psychol. 33, 649-665. doi: 10.1123/jsep.33.5.649

Hüttermann, S., and Memmert, D. (2014). Does the inverted-U function disappear in expert athletes? An analysis of the attentional behavior under physical exercise of athletes and non-athletes. Physiol. Behav. 131, 87-92. doi: 10.1016/j. physbeh.2014.04.020

Hyland-Monks, R., Cronin, L., McNaughton, L., and Marchant, D. (2018). The role of executive function in the self-regulation of endurance performance: a critical review. Prog. Brain Res. 240, 353-370. doi: 10.1016/bs.pbr.2018.09.011

John, D., Bassett, D., Thompson, D., Fairbrother, J., and Baldwin, D. (2009). Effect of using a treadmill workstation on performance of simulated office work tasks. J. Phys. Act. Health 6, 617-624. doi: 10.1123/jpah.6.5.617

Joyce, J., Graydon, J., McMorris, T., and Davranche, K. (2009). The time course effect of moderate intensity exercise on response execution and response inhibition. Brain Cogn. 71, 14-19. doi: 10.1016/j.bandc.2009.03.004

Joyce, J., Smyth, P. J., Donnelly, A. E., and Davranche, K. (2014). The simon task and aging: does acute moderate exercise influence cognitive control? Med. Sci. Sports Exerc. 46, 630-639. doi: 10.1249/MSS.0b013e3182a77980

Kaminsky, L. A., Arena, R., and Myers, J. (2015). Reference standards for cardiorespiratory fitness measured with cardiopulmonary exercise testing: data from the fitness registry and the importance of exercise national database. Mayo Clin. Proc. 90, 1515-1523. doi: 10.1016/j.mayocp.2015.07.026

Keyan, D., and Bryant, R. A. (2017b). Brief exercise enhances intrusive memories of traumatic stimuli. Neurobiol. Learn. Mem. 141, 9-13. doi: 10.1016/j.nlm.2017. 03.012

Keyan, D., and Bryant, R. A. (2017a). Acute physical exercise in humans enhances reconsolidation of emotional memories. Psychoneuroendocrinology 86, 144151. doi: 10.1016/j.psyneuen.2017.09.019

Komiyama, T., Katayama, K., Sudo, M., Ishida, K., Higaki, Y., and Ando, S. (2017). Cognitive function during exercise under severe hypoxia. Sci. Rep. 7, 1-11. doi: 10.1038/s41598-017-10332-y

Komiyama, T., Sudo, M., Costello, J. T., and Uehara, Y. (2019). Cognitive impairment during high-intensity exercise: influence of cerebral blood flow. Med. Sci. Sports Exerc. 52, 561-568. doi: 10.1249/MSS.0000000000002183

Komiyama, T., Sudo, M., Higaki, Y., Kiyonaga, A., Tanaka, H., and Ando, S. (2015). Does moderate hypoxia alter working memory and executive function during prolonged exercise? Physiol. Behav. 139, 290-296. doi: 10.1016/J.PHYSBEH. 2014.11.057

Komiyama, T., Sudo, M., Okuda, N., Yasuno, T., Kiyonaga, A., Tanaka, H., et al. (2016). Cognitive function at rest and during exercise following breakfast omission. Physiol. Behav. 157, 178-184. doi: 10.1016/j.physbeh.2016.02.013

Labban, J. D., and Etnier, J. L. (2011). Effects of acute exercise on long-term memory. Res. Q. Exerc. Sport 82, 712-721. doi: 10.1080/02701367.2011. 10599808

Labelle, V. V. V., Bosquet, L., Mekary, S. S. S., and Bherer, L. (2013). Decline in executive control during acute bouts of exercise as a function of exercise 
intensity and fitness level. Brain Cogn. 81, 10-17. doi: 10.1016/j.bandc.2012. 10.001

Lambourne, K., Audiffren, M., and Tomporowski, P. D. (2010). Effects of acute exercise on sensory and executive processing tasks. Med. Sci. Sports Exerc. 42, 1396-1402. doi: 10.1249/MSS.0b013e3181cbee11

Lambourne, K., and Tomporowski, P. (2010). The effect of exercise-induced arousal on cognitive task performance: a meta-regression analysis. Brain Res. 1341, 12-24. doi: 10.1016/j.brainres.2010.03.091

Lichtenberger, E., and Kaufman, A. (2009). Essentials of WAIS-IV Assessment. Hoboken, NJ: John Wiley \& Sons, Inc.

Logan, G. D., and Cowan, W. B. (1984). On the ability to inhibit thought and action: a theory of an act of control. Psychol. Rev. 91, 295-295. doi: 10.1037/a0035230

Loprinzi, P. D. (2018). Intensity-specific effects of acute exercise on human memory function: considerations for the timing of exercise and the type of memory. Health Promot. Perspect. 8, 255-262. doi: 10.15171/hpp.2018.36

Loprinzi, P. D. (2019). An integrated model of acute exercise on memory function. Med. Hypotheses 126, 51-59. doi: 10.1016/J.MEHY.2019.03.010

Loprinzi, P. D., Day, S., Hendry, R., Hoffman, S., Love, A., Marable, S., et al. (2021). The effects of acute exercise on short-and long-term memory: considerations for the timing of exercise and phases of memory. Eur. J. Psychol. 17, 85-103. doi: $10.5964 /$ ejop. 2955

Loprinzi, P. D., Lovorn, A., and Gilmore, J. (2020). Effects of exercise on explicit memory function: incidental and intentional encoding may depend on exercise timing. Percept. Mot. Skills 128, 865-884. doi: 10.1177/0031512520979671

McMorris, T. (2016). "Exercise-cognition interaction: state of the art and future research," in Exercise-Cognition Interaction: Neuroscience Perspectives, ed. T. McMorris (Cambridge, MA: Elsevier Academic Press). doi: 10.1016/B978-012-800778-5.00022-0

McMorris, T., Collard, K., Corbett, J., Dicks, M., and Swain, J. P. (2008). A test of the catecholamines hypothesis for an acute exercise-cognition interaction. Pharmacol. Biochem. Behav. 89, 106-115. doi: 10.1016/j.pbb.2007.11.007

McMorris, T., Davranche, K., Jones, G., Hall, B., Corbett, J., and Minter, C. (2009). Acute incremental exercise, performance of a central executive task, and sympathoadrenal system and hypothalamic-pituitary-adrenal axis activity. Int. J. Psychophysiol. 73, 334-340. doi: 10.1016/j.ijpsycho.2009.05.004

McMorris, T., and Graydon, J. (1997). The effect of exercise on cognitive performance in soccer-specific tests. J. Sports Sci. 15, 459-468. doi: 10.1080/ 026404197367092

McMorris, T., and Graydon, J. (2000). The effect of incremental exercise on cognitive performance. Int. J. Sport Psychol. 31, 66-81.

McMorris, T., and Hale, B. J. (2012). Differential effects of differing intensities of acute exercise on speed and accuracy of cognition: a meta-analytical investigation. Brain Cogn. 80, 338-351. doi: 10.1016/j.bandc.2012.09.001

McMorris, T., and Hale, B. J. (2015). Is there an acute exercise-induced physiological/biochemical threshold which triggers increased speed of cognitive functioning? A meta-analytic investigation. J. Sport Health Sci. 4, 4-13. doi: 10.1016/j.jshs.2014.08.003

McMorris, T., Sproule, J., Turner, A., and Hale, B. J. (2011). Acute, intermediate intensity exercise, and speed and accuracy in working memory tasks: a metaanalytical comparison of effects. Physiol. Behav. 102, 421-428. doi: 10.1016/J. PHYSBEH.2010.12.007

McMorris, T., Turner, A., Hale, B. J., and Sproule, J. (2016). "Beyond the catecholamines hypothesis for an acute exercise-cognition interaction: a neurochemical perspective," in Exercise-Cognition Interaction: Neuroscience Perspectives, ed. T. McMorris (Cambridge, MA: Elsevier Academic Press), 65-103. doi: 10.1016/b978-0-12-800778-5.00004-9

Miles, C., and Hardman, E. (1998). State-dependent memory produced by aerobic exercise. Ergonomics 41, 20-28. doi: 10.1080/001401398187297

Miyake, A., and Friedman, N. (2012). The nature and organization of individual differences in executive functions: four general conclusions. Curr. Dir. Psychol. Sci. 21, 8-14. doi: 10.1177/0963721411429458.The

Miyake, A., Friedman, N. P., Emerson, M. J., Witzki, A. H., Howerter, A., and Wager, T. D. (2000). The unity and diversity of executive functions and their contributions to complex "Frontal Lobe" tasks: a latent variable analysis. Cogn Psychol. 41, 49-100. doi: 10.1006/cogp.1999.0734

Miyake, A., and Shah, P. (1999). Models of Working Memory: Mechanisms of Active Maintenance and Executive Control. Cambridge, MA: Cambridge University Press.
Navon, D. (1977). Forest before trees: the precedence of global features in visual perception. Cogn. Psychol. 9, 353-353. doi: 10.1016/0010-0285(77)90 012-3

Nigg, J. T. (2017). Annual research review: on the relations among self-regulation, self-control, executive functioning, effortful control, cognitive control, impulsivity, risk-taking, and inhibition for developmental psychopathology. J. Child Psychol. Psychiatry 58, 361-383. doi: 10.1111/jcpp. 12675

Olson, R. L., Chang, Y. K., Brush, C. J., Kwok, A. N., Gordon, V. X., and Alderman, B. L. (2016). Neurophysiological and behavioral correlates of cognitive control during low and moderate intensity exercise. NeuroImage 131, 171-180. doi: 10.1016/j.neuroimage.2015.10.011

Oppezzo, M., and Schwartz, D. L. (2014). Give your ideas some legs: the positive effect of walking on creative thinking. J. Exp. Psychol. 40, 1142-1152. doi: $10.1037 / \mathrm{a} 0036577$

Paas, F., and Adam, J. J. J. (1991). Human information processing during physical exercise. Ergonomics 34, 1385-1397. doi: 10.1080/00140139108964879

Pesce, C., Capranica, L., Tessitore, A., and Figura, F. (2002). Effects of a submaximal physical load on the orienting and focusing of visual attention. J. Hum. Mov. Stud. 42, 401-420.

Pesce, C., Capranica, L., Tessitore, A., and Figura, F. (2003). Focusing of visual attention under submaximal physical load. Int. J. Sport Exerc. Psychol. 1, 275-292. doi: 10.1080/1612197x.2003.9671719

Pesce, C., Casella, R., and Capranica, L. (2004). Modulation of visuospatial attention at rest and during physical exercise: gender differences. Int. J. Sport Psychol. 35, 328-341.

Pesce, C., Tessitore, A., Casella, R., Pirritano, M., and Capranica, L. (2007). Focusing of visual attention at rest and during physical exercise in soccer players. J. Sports Sci. 25, 1259-1270. doi: 10.1080/026404106010 40085

Piercy, K. L., Troiano, R. P., Ballard, R. M., Carlson, S. A., Fulton, J. E., Galuska, D. A., et al. (2018). The physical activity guidelines for Americans. JAMA 320, 2020-2028. doi: 10.1001/jama.2018.14854

Pontifex, M., and Hillman, C. H. (2007). Neuroelectric and behavioral indices of interference control during acute cycling. Clin. Neurophysiol. 118, 570-580. doi: 10.1016/j.clinph.2006.09.029

Pontifex, M., McGowan, A. L., Chandler, M. C., Gwizdala, K. L., Parks, A. C., Fenn, K., et al. (2019). A primer on investigating the after effects of acute bouts of physical activity on cognition. Psychol. Sport Exerc. 40, 1-22. doi: 10.1016/j.psychsport.2018.08.015

Pyke, W., Ifram, F., Coventry, L., Sung, Y., Champion, I., and Javadi, A. H. (2020). The effects of different protocols of physical exercise and rest on longterm memory. Neurobiol. Learn. Mem. 167, 1-8. doi: 10.1016/j.nlm.2019.10 7128

Quelhas Martins, A., Kavussanu, M., Willoughby, A., and Ring, C. (2013). Moderate intensity exercise facilitates working memory. Psychol. Sport Exerc. 14, 323-328. doi: 10.1016/j.psychsport.2012.11.010

Radel, R., Brisswalter, J., and Perrey, S. (2017). Saving mental effort to maintain physical effort: a shift of activity within the prefrontal cortex in anticipation of prolonged exercise. Cogn. Affect. Behav. Neurosci. 17, 305-314. doi: 10.3758/ s13415-016-0480-x

Radel, R., Tempest, G. D., and Brisswalter, J. (2018). The long and winding road: effects of exercise intensity and type upon sustained attention. Physiol. Behavi. 195, 82-89. doi: 10.1016/j.physbeh.2018.07.028

Rattray, B., and Smee, D. (2013). Exercise improves reaction time without compromising accuracy in a novel easy-to-administer tablet-based cognitive task. J. Sci. Med. Sport 16, 567-570. doi: 10.1016/J.JSAMS.2012. 12.007

Rattray, B., and Smee, D. J. (2016). The effect of high and low exercise intensity periods on a simple memory recognition test. J. Sport Health Sci. 5, 342-348. doi: 10.1016/j.jshs.2015.01.005

Roig, M., Nordbrandt, S., Geertsen, S. S., and Nielsen, J. B. (2013). The effects of cardiovascular exercise on human memory: a review with meta-analysis. Neurosci. Biobehavi. Rev. 37, 1645-1666. doi: 10.1016/J.NEUBIOREV.2013.06. 012

Sanabria, D., Morales, E., Luque, A., Gálvez, G., Huertas, F., and Lupiañez, J. (2011). Effects of acute aerobic exercise on exogenous spatial attention. Psychol. Sport Exerc. 12, 570-574. doi: 10.1016/J.PSYCHSPORT.2011. 04.002 
Sanchis, C., Blasco, E., Luna, F. G., and Lupiáñez, J. (2020). Effects of caffeine intake and exercise intensity on executive and arousal vigilance. Sci. Rep. 10:8393. doi: 10.1038/s41598-020-65197-5

Schmit, C., and Brisswalter, J. (2018). Executive functioning during prolonged exercise: a fatigue-based neurocognitive perspective. Int. Rev. Sport Exerc. Psychol. 13, 1-19. doi: 10.1080/1750984x.2018.1483527

Schmit, C., Davranche, K., Easthope, C. S., Colson, S. S., Brisswalter, J., and Radel, R. (2015). Pushing to the limits: the dynamics of cognitive control during exhausting exercise. Neuropsychologia 68, 71-81. doi: 10.1016/j. neuropsychologia.2015.01.006

Shields, M. R., Larson, C. L., Swartz, A. M., and Smith, J. C. (2011). Visual threat detection during moderate- and high-intensity exercise. Emotion 11, 572-581. doi: 10.1037/a0021251

Silvers, W. M., Newby, T. A., and Parker, J. K. (2018). The effects of different aerobic exercise intensities on short-term and long-term memory retention following a video-based learning task: a pilot study. J. Kinesiol. Wellness 7, 17-28.

Simon, J. R., and Wolf, J. D. (1963). Choice reaction time as a function of angular stimulus-response correspondence and age. Ergonomics 6, 99-105. doi: 10. 1080/00140136308930679

Smith, M., Tallis, J., Miller, A., Clarke, N. D., Guimarães-Ferreira, L., and Duncan, M. J. (2016). The effect of exercise intensity on cognitive performance during short duration treadmill running. J. Hum. Kinet. 50, 27-35. doi: 10.1515/hukin2015-0167

Stroop, J. R. (1935). Studies of interference in serial verbal reactions. J. Exp. Psychol. 18, 643-662. doi: 10.1037/h0054651

Tempest, G. D., Davranche, K., Brisswalter, J., Perrey, S., and Radel, R. (2017). The differential effects of prolonged exercise upon executive function and cerebral oxygenation. Brain Cogn. 113, 133-141. doi: 10.1016/J.BANDC.2017. 02.001

Tenenbaum, G. (2007). Decision in the form of attention: the speedaccuracy trade-off and the cost-benefit paradigm. J. Sport Exerc. Psychol. 29, S11-S11.

Tomporowski, P. D. (2003). Effects of acute bouts of exercise on cognition. Acta Psychol. 112, 297-324. doi: 10.1016/s0001-6918(02)00134-8

Travlos, A. K., and Marisi, D. Q. (1995). Information processing and concentration as a function of fitness level and exercise-induced activation to exhaustion. Percept. Mot. Skills 80, 15-26. doi: 10.2466/pms.1995.80.1.15

van Dongen, E. V., Kersten, I. H. P., Wagner, I. C., Morris, R. G. M., and Fernández, G. N. (2016). Physical exercise performed four hours after learning improves memory retention and increases hippocampal pattern similarity during retrieval. Curr. Biol. 26, 1722-1727. doi: 10.1016/j.cub.2016.04.071

Wang, C. C., Chu, C. H., Chu, I. H., Chan, K. H., and Chang, Y. K. (2013). Executive function during acute exercise: the role of exercise intensity. J. Sport Exerc. Psychol. 35, 358-367.

Wang, X., Zhu, R., Zhou, C., and Chen, Y. (2020). Distinct effects of acute aerobic exercise on declarative memory and procedural memory formation. Brain Sci. 10, 1-51. doi: 10.3390/brainsci10100691

Wingfield, G., Marino, F. E., and Skein, M. (2019). Deception of cycling distance on pacing strategies, perceptual responses, and neural activity. Pflügers Arch. Eur. J. Physiol. 471, 285-299. doi: 10.1007/s00424-018-2218-9

Wohlwend, M., Olsen, A., Håberg, A. K., and Palmer, H. S. (2017). Exercise intensity-dependent effects on cognitive control function during and after acute treadmill running in young healthy adults. Front. Psychol. 8:406. doi: 10.3389/ fpsyg.2017.00406

Yagi, Y., Coburn, K. L., Estes, K. M., and Arruda, J. E. (1999). Effects of aerobic exercise and gender on visual and auditory P300, reaction time, and accuracy. Eur. J. Appl. Physiol. Occup. Physiol. 80, 402-408. doi: 10.1007/s0042100 50611

Yerkes, R. M., and Dodson, J. D. (1908). The relationship of strength of stimulus to rapidity of habit formation. J. Comp. Neurol. Psychol. 18, 459-482. doi: $10.1002 /$ cne. 920180503

Conflict of Interest: The authors declare that the research was conducted in the absence of any commercial or financial relationships that could be construed as a potential conflict of interest.

Publisher's Note: All claims expressed in this article are solely those of the authors and do not necessarily represent those of their affiliated organizations, or those of the publisher, the editors and the reviewers. Any product that may be evaluated in this article, or claim that may be made by its manufacturer, is not guaranteed or endorsed by the publisher.

Copyright (C) 2021 Cantelon and Giles. This is an open-access article distributed under the terms of the Creative Commons Attribution License (CC BY). The use, distribution or reproduction in other forums is permitted, provided the original author(s) and the copyright owner(s) are credited and that the original publication in this journal is cited, in accordance with accepted academic practice. No use, distribution or reproduction is permitted which does not comply with these terms. 